\title{
The Physics of Accretion Discs, Winds And Jets in Tidal Disruption Events
}

\author{
Jane Lixin Dai · Giuseppe Lodato • \\ Roseanne Cheng
}

Received: date / Accepted: date

\begin{abstract}
Accretion onto black holes is an efficient mechanism in converting the gas mass-energy into energetic outputs as radiation, wind and jet. Tidal disruption events, in which stars are tidally torn apart and then accreted onto supermassive black holes, offer unique opportunities of studying the accretion physics as well as the wind and jet launching physics across different accretion regimes. In this review, we systematically describe and discuss the models that have been developed to study the accretion flows and jets in tidal disruption events. A good knowledge of these physics is not only needed for understanding the emissions of the observed events, but also crucial for probing the general relativistic space-time around black holes and the demographics of supermassive black holes via tidal disruption events.
\end{abstract}

Keywords accretion, accretion discs - black hole physics · galaxies: jets · galaxies: nuclei $\cdot$ gravitation $\cdot$ hydrodynamics

\footnotetext{
J. L. Dai

Department of Physics

The University of Hong Kong

Pokfulam Road, Hong Kong (HK)

E-mail: lixindai@hku.hk

G. Lodato

Università degli Studi di Milano

Dpartimento di Fisica

Via Celoria 16, Milano (Italy)

E-mail: giuseppe.lodato@unimi.it

R. M. Cheng

Theoretical Division (T-3)

Los Alamos National Laboratory

P.O. Box 1663

Los Alamos, NM 87545 (USA)

E-mail: rmcheng@lanl.gov
} 


\section{Introduction}

A tidal disruption event (TDE) happens when a star passes by a supermassive black hole (SMBH) too close and is therefore torn apart by the tidal force. The distance at which the SMBH tidal force equals the stellar self-gravity is called the tidal radius $R_{t}$ (Hills 1975), which depends on the mass $M_{\star}$ and radius $R_{\star}$ of the star as well as the mass $M_{\mathrm{BH}}$ of the SMBH: $R_{t} \approx R_{\star}\left(M_{\mathrm{BH}} / M_{\star}\right)^{1 / 3}$. For main-sequence stars approaching SMBHs with $M_{\mathrm{BH}}=$ few $\times\left(10^{6}-10^{7}\right) M_{\odot}$, $R_{t}$ is only few $\times(1-10) R_{g}$ with $R_{g} \equiv G M_{\mathrm{BH}} / c^{2}$ being the gravitational radius of the black hole. Therefore, general relativity (GR) naturally plays an important role in the processes happening in TDEs.

The basic theory of TDE physics has been developed since the 1970s (e.g., Hills 1975; Rees 1988; Phinney 1989). Here we give a brief description of the general picture. Following the disruption of a star approaching the black hole along a marginally bound orbit, roughly half of the stellar debris remains bound and travels along highly elliptical, ballistic orbits, while the other half escapes along hyperbolic orbits. The spread of the orbital energy determines the mass rate that bound debris returns to the pericenter as a function of time, which is called the fallback rate. Shortly after passing through the pericenter, the bound debris assembles a transient accretion disc around the SMBH through shocks and shear viscosity reducing the orbital energy of the debris. Then with the aid of magnetic fields, viscous processes transport the gas angular momentum outwards, allowing gas to accrete onto the black hole and bright flares to be produced. Under optimal conditions, winds and relativistic jets can also be launched from the transient discs formed in TDEs.

In recent years our understanding of TDE physics has been greatly pushed forward by numerical simulations. These simulations usually focus on specific phases in TDEs (e.g., the disruption of the star, the evolution of the debris stream, the formation of the disc, the accretion process and jet production, etc.) due to the large computational resources required to study the whole process. These studies have basically confirmed the disruption process described in the general picture, which is discussed in the Disruption Chapter of this book. However, up to now no self-consistent simulations have been done on the disc formation process for typical TDE parameters, leading to uncertainties on whether the debris can quickly dissipate orbital energy to form discs as described in the general picture. We refer the readers to the Formation of the Accretion Flow Chapter for details. In this Chapter dedicated to the TDE accretion process, we will focus on the phase after stable discs have been formed and review the theoretical efforts that have been made to investigate the physics of TDE accretion discs, winds and jets.

A solid understanding of the accretion process builds foundations for understanding TDE emissions, and therefore is key for probing SMBH demographics from TDE observables. Also, TDEs give us ideal laboratories for studying black hole accretion physics in different accretion regimes. In a standard TDE (where a main-sequence star is totally disrupted by a SMBH along a parabolic orbit), the debris fallback rate that feeds the disc is expected to first quickly 
reach the super-Eddington regime, and then declines to the sub-Eddington regime over a few months to years (Fig. 1).

The Eddington luminosity is the theoretical maximum luminosity that a star or an accretion disc can have when there is a balance between the outward radiation force and the inward gravitational force. For the inner part of accretion discs, all of the hydrogen atoms are ionized since the temperature is higher than $10^{5} \mathrm{~K}$ (Shakura and Sunyaev 1973; Frank et al. 2002). The Eddington luminosity for a black hole is therefore defined as:

$$
L_{\mathrm{Edd}}=\frac{4 \pi c G M_{\mathrm{BH}}}{\kappa_{\mathrm{es}}} \approx 1.26 \times 10^{44}\left(\frac{M_{\mathrm{BH}}}{10^{6} M_{\odot}}\right) \mathrm{erg} \mathrm{s}^{-1} .
$$

Here $\kappa_{\mathrm{es}}$ is the Thomson scattering opacity. For hydrogen $\kappa_{\mathrm{es}}=\sigma_{\mathrm{T}} / m_{\mathrm{p}}=$ $0.4 \mathrm{~cm}^{2} \mathrm{~g}^{-1}$, where $m_{\mathrm{p}}$ is the proton mass and $\sigma_{\mathrm{T}}$ is the Thomson cross-section. The corresponding Eddington accretion rate is:

$$
\dot{M}_{\mathrm{Edd}}=\frac{L_{\mathrm{Edd}}}{\eta c^{2}} \approx 0.022\left(\frac{\eta}{0.1}\right)^{-1}\left(\frac{M}{10^{6} M_{\odot}}\right) M_{\odot} \mathrm{yr}^{-1},
$$

where $\eta \equiv L /\left(\dot{M} c^{2}\right)$ is the accretion radiative efficiency. In literature $\eta$ is usually given a nominal value of $10 \%$, although its value can vary drastically as described next.

The structure of an accretion disc is primarily determined by its Eddington ratio, which is how the accretion rate compares to the Eddington accretion rate. (1) For very high, super-Eddington rates $\left(\dot{M} / \dot{M}_{\mathrm{Edd}} \gtrsim 1\right)$, the disc is expected to be geometrically and optically thick. The photon diffusion time is very long and the disc is dominated by advection (the so-called slim disc solutions by Abramowicz et al. 1988) resulting in low radiative efficiency; (2) for moderate accretion rates, $0.01 \lesssim \dot{M} / \dot{M}_{\text {Edd }} \lesssim 1$ (or $0.01 \lesssim L / L_{\text {Edd }} \lesssim 0.3$ ), the disc, well described by the standard Shakura and Sunyaev (1973) solutions (or a GR prescription by Novikov and Thorne 1973), is optically thick, geometrically thin, and radiatively efficient; finally, (3) for very low accretion rates $\left(\dot{M} / \dot{M}_{\text {Edd }}<0.01\right)$, the disc transitions again to an advection-dominated accretion flow (ADAF) or a radiatively inefficient accretion flow (RIAF) (Ichimaru 1977; Rees et al. 1982; Narayan and Yi 1994; Blandford and Begelman 1999, 2004) with low radiative efficiency again. Although the Eddington ratio likely plays the most important role in determining the morphology of an accretion disc, the properties of the disc are also affected other physical parameters. For example, the black hole mass, together with the accretion rate, determine the disc temperature and various opacities governing disc dynamics and radiative processes. Also, in the thin disc regime, the inner edge of the disc is set by the innermost stable circular orbit (ISCO) which has a strong dependence on the black hole spin. As a result, $\eta$ varies from $6 \%$ to $42 \%$ as the black hole spin increases from 0 to 1.

Ulmer (1999) is the first to point out that different regimes of accretion should naturally happen throughout the course of a TDE, so the disc geometry should change from thick to thin as the accretion level drops from superEddington to sub-Eddington in a few months to years. Analytical models that 


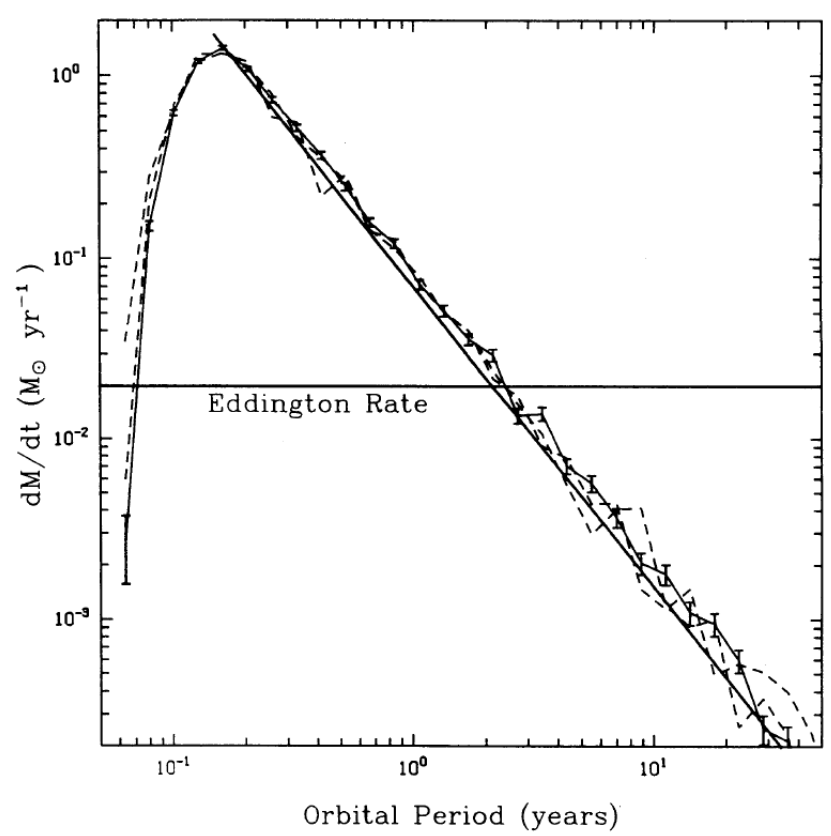

Fig. 1 The debris fallback rate as a function of time. The Eddington accretion rate shown is for a $\mathrm{SMBH}$ with $M_{\mathrm{BH}}=10^{6} M_{\odot}$ and radiative efficiency $\eta=0.1$. As shown the fallback rate can be one to two orders of magnitude above the Eddington accretion rate of the black hole when $M_{\mathrm{BH}}<$ few $\times 10^{7} M_{\odot}$. After the peak the fallback rate declines following a powerlaw function of time: $\dot{M}_{\mathrm{fb}} \propto t^{-5 / 3}$, sweeping through different regimes of accretion. Figure is from Evans and Kochanek (1989).

encompass both the slim disc case and the standard thin disc case have been first constructed by Strubbe and Quataert (2009). We will review various disc models and simulations along this line in Section 2.1 (early, super-Eddington phase) and Section 2.2 (late time). New disc models or angular momentum transport mechanisms different from the conventional picture will be reviewed in Section 2.3 and Section 2.4.

Relativistic jets and winds have been observed in several TDEs, which cannot been explained using the standard thin disc theory. In Section 2.1.2 and Section 3, we will review works which discuss how winds and jets can be launched when TDE disc is in the super-Eddington accretion regime. A cartoon illustrating the disc-wind-jet geometry during this phase is illustrated in Fig. 2.

Most TDE literature have so far focused on modeling discs which have angular momentum aligned with the spin axis of the black hole. However, misaligned TDE discs likely can form since it is generally expected that stars are scattered into the loss cone from random orientations. We will discuss works that have been carried out to understand such discs and their jets in Section 4. 


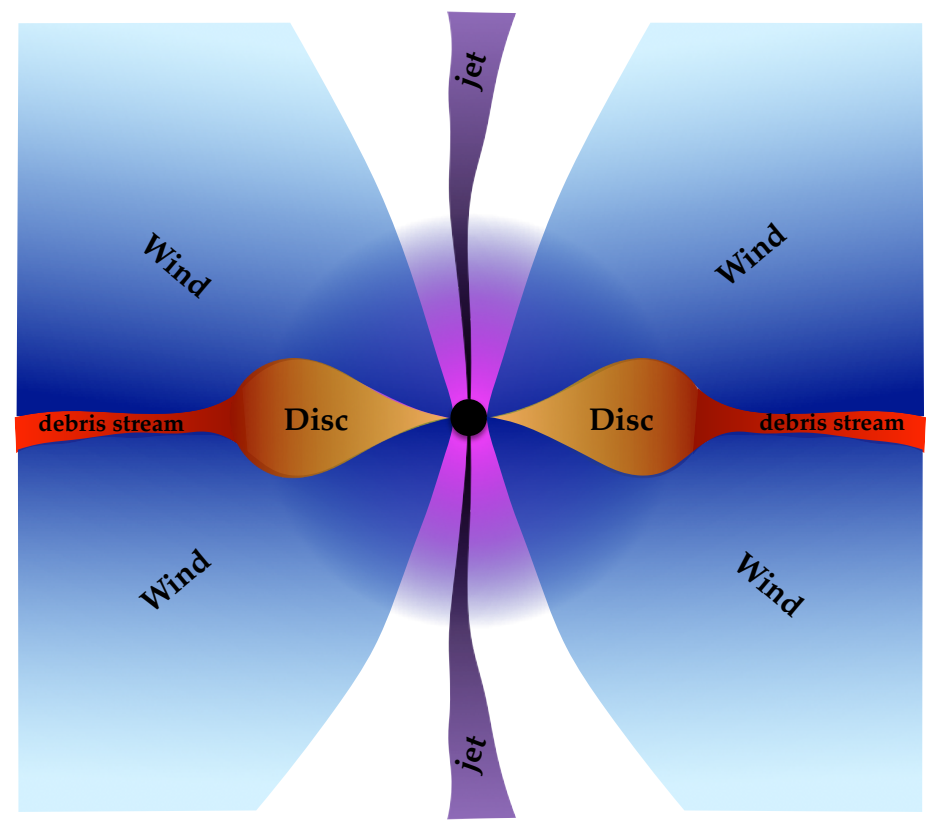

Fig. 2 A cartoon showing the geometry of a circular and aligned geometrically-thick accretion disc formed in the super-Eddington phase of a TDE, as well as the wind and jet launched from the disc. It is expected that a super-Eddington disc can generally launch optically-thick wind with large solid angle coverage. A strong relativistic jet, on the other hand, can only be produced when the black hole is spinning fast and has a large amount of ordered magnetic flux in its vicinity (Blandford and Znajek 1977). Figure is adapted from Dai et al. (2018).

Lastly, a summary will be drawn in Section 5, where we will also raise several key open questions related to TDE accretion discs, winds and jets for future studies.

\section{Models of TDE Accretion Flow}

In this section, we will summarize various analytical models and numerical simulations that have been carried out to study the accretion flow formed in TDEs. While we put the focus on describing the structures of the accretion inflow/outflow and the accretion process, we will also briefly mention the properties of the emissions produced in these models. We refer the readers to the Emission Mechanisms Chapter in this book for more details of the radiative processes in TDE accretion flow. 
2.1 TDE discs with super-Eddington debris fallback

During the first few weeks to years in a TDE, the debris supply rate is high and can exceed the Eddington accretion rate of the black hole if the black hole mass $M_{\mathrm{BH}} \lesssim$ few $\times 10^{7} M_{\odot}$. It is naturally expected that most TDEs are detected in this phase as the flares are luminous. Many theoretical studies have therefore focused on studying the properties of the TDE discs in this phase. Most of the work are semi-analytical, in which the TDE accretion flow is modeled as a steady, quasi-spherical disc (Loeb and Ulmer 1997; Ulmer 1999; Coughlin and Begelman 2014; Roth et al. 2016; Wu et al. 2018) or a disc surrounded by a quasi-spherical wind (Strubbe and Quataert 2009; Lodato and Rossi 2011; Shen and Matzner 2014; Miller 2015; Metzger and Stone 2016). Recently 3D numerical simulations of super-Eddington accretion flow have also been carried out to study TDE discs, in which the disc winds (and jets) are self-consistently produced (Dai et al. 2018; Curd and Narayan 2019).

From the observational side, the unique properties of TDE emissions also lend strong support to the notion that TDE discs should be different from the discs of local AGNs, which are believed to be sub-Eddington in most cases. Some of the most relevant observational features are: 1) Many TDE flares are strong in UV/optical wavebands but weak in X-rays, while some TDEs are strong in X-rays but not in UV/optical bands. 2) For the optically-strong TDEs, their flares are usually bluer than AGN optical emissions. There are three spectroscopic classes discovered: TDEs that produce broad H Balmer emission lines, He emission lines, or Bowen fluorescent NIII/OIII emission lines (Gezari et al. 2012; Arcavi et al. 2014; Leloudas et al. 2019; van Velzen et al. 2020). 3) For the non-jetted X-ray strong TDEs, their emissions are dominated by thermal components and do not show strong coronae which are typically seen in AGNs (Komossa 2015; Auchettl et al. 2017). 4) In some TDEs, fast winds have been reported, which will be discussed more in Section 2.1.2. More details on how TDE emissions are distinctive from AGN emissions can be found in the Imposters Chapter. The disc models to be discussed in this section all employ extreme debris supply or accretion level to explain these observed TDE features.

\subsubsection{Quasi-spherical models of steady discs or envelopes}

Early studies (e.g., Rees 1988) assume that stellar debris can circularize fast and form a compact disc with a size $R_{c}=2 R_{p}$ from conservation of angular momentum, where $R_{p}$ is the pericenter radius of the original parabolic orbit of the star. Due to the short viscous timescale of the disc, the debris accretion rate resembles the fallback rate. Therefore, for the first few weeks to months, the disc in the super-Eddington accretion regime is geometrically and optically thick. Ulmer (1999) calculates the effective temperature of this small, quasi-spherical disc by assuming the luminosity is limited by the Eddington 


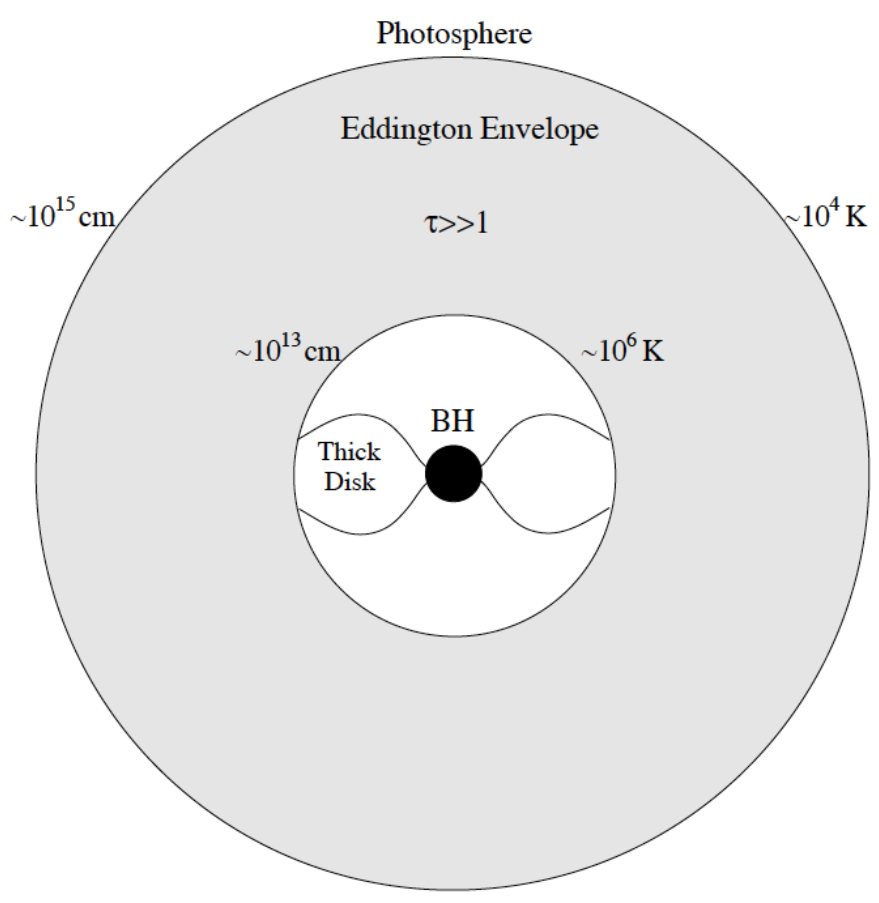

Fig. 3 Illustration of a steady, optically-thick, quasi-spherical large envelope surrounding a small accretion disc formed in TDEs. The disc X-ray/EUV emissions are reprocessed into $\mathrm{UV} /$ optical emissions when going through the envelope. Figure is from Loeb and Ulmer (1997).

luminosity $L_{\mathrm{Edd}}$ and the disc radius is comparable to the tidal radius $R_{t}$ :

$$
T_{\text {eff }}=\left(\frac{L_{\mathrm{Edd}}}{4 \pi R_{t}^{2} \sigma}\right) \approx 5 \times 10^{5} M_{6}^{-1 / 4} \mathrm{~K},
$$

where $\sigma$ is the Stefan-Boltzmann constant and $M_{6}=M_{\mathrm{BH}} /\left(10^{6} M_{\odot}\right)$.

This simple model predicts that TDEs should shine in X-ray/EUV and can therefore explain the observed X-ray strong TDEs (see the X-ray Chapter of this book). However, it cannot explain why many TDEs are only bright in optical/NUV bands but weak in X-rays (see the Optical Chapter). It has been proposed by Loeb and Ulmer (1997) that since the stellar debris is only marginally bound, radiation produced from the viscous process in the inner accretion disc can easily disperse some gas into a quasi-spherical configuration at a large distance. This large, steady, optically-thick envelope surrounding the small accretion disc, as illustrated in Fig. 3, can reprocess the disc X-ray/EUV emissions into optical emissions. The envelope is supported by radiation pressure and has a density profile of $\rho(r) \propto r^{-q}$ with $q=3$. It is assumed to be in a steady state exactly balanced by gravity and radiation pressure, which relies 


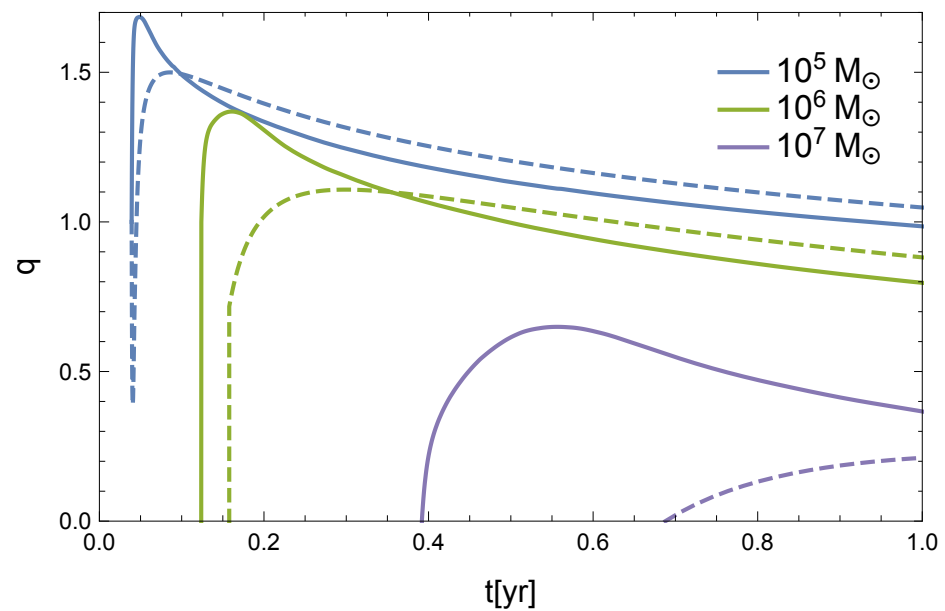

Fig. 4 Index $q$ of the zero-Bernoulli accretion (ZEBRA) flow (Coughlin and Begelman 2014) density profile $\left(\rho(r) \propto r^{-q}\right)$ as a function of time for TDEs around black holes of different masses. The solid lines show results using numerically acquired fallback rate, and the dashed lines show results using the "frozen-in" approximation at the time of disruption. Figure is from $\mathrm{Wu}$ et al. (2018).

on a super-Eddington accretion rate with a radiative efficiency of $\gtrsim 10^{-3}$. The photosphere shines at the Eddington luminosity with a size of about $10^{15} \mathrm{~cm}$ and an effective temperature of around $10^{4} \mathrm{~K}$.

Later Coughlin and Begelman (2014) argue that the angular momentum transport between the disc and envelope in the setting of Loeb and Ulmer (1997) should further shrink the disc and flatten its density distribution, making it hard to maintain the accretion exactly at the Eddington level. Instead, they propose a model in which the whole accretion disc is inflated by radiation energy into a quasi-spherical configuration with zero Bernoulli parameter (i.e., marginally bound) everywhere (these models are called "Zero-Bernoulli accretion flows", or ZEBRAs). The disc rotates at a sub-Keplerian speed with a very small angular momentum consistent with the angular momentum of the returning stellar debris. The evolution of the accretion flow has been computed using the analytical debris fallback rate derived from the "frozen-in" approximation (Lodato et al. 2009) as well as using the numerically computed fallback rate (Wu et al. 2018). As shown in Fig. 4, the density profile of the accretion flow $\rho(r) \propto r^{-q}$ has an index $q$ ranging between 0.5 and 1.5 during most events. The photosphere radius grows with time to $R_{\mathrm{ph}} \sim$ few $\times 10^{14} \mathrm{~cm}$ and the photosphere temperature drops by a few times to $T_{\mathrm{ph}} \sim$ few $\times 10^{4} \mathrm{~K}$ after a few years. In this model, most of the energy produced from accretion is actually released in the form of a jet along polar directions confined by the narrow rotational funnel. 
This type of spherical and steady envelope model has also been adopted by e.g., Roth et al. (2016) to carry out detailed radiative transfer studies in TDEs.

\subsubsection{Models of TDE discs with winds}

A small fraction of the energy produced through accretion or other energetic processes (such as shock heating during disc formation) can in principle unbind a large fraction of returning debris and launch strong winds. Wind-launching from accretion discs can be due to: 1) large radiation pressure in super-Eddington accretion discs or other radiative transfer processes in accretion discs (e.g., Poutanen et al. 2007; Proga et al. 2000; Dotan and Shaviv 2011); 2) hydromagnetic processes (e.g. Blandford and Payne 1982); 3) in TDEs, gas heating during the processes leading to disc formation such as collisions of debris steams (Jiang et al. 2016).

Winds have been observed in several TDEs. For example, ultra-fast winds with $v \gtrsim 0.1 c$ have been found through $\mathrm{X}$-ray reflection signatures in Swift J1644 (Kara et al. 2016) and through X-ray absorption features or radio signals found in (soft) X-ray strong TDE ASASSN 14-li (Alexander et al. 2016; Kara et al. 2017) and 3XMM J1500 (Lin et al. 2017). Recently UV spectroscopic studies have also been fruitful in discovering winds with $v \approx 0.05 \mathrm{c}$ from optical strong TDEs (Hung et al. 2019).

In the context of TDE discs, there are several studies modeling or simulating the winds launched during the super-Eddington accretion phase (Strubbe and Quataert 2009, 2011; Lodato and Rossi 2011; Dai et al. 2018; Curd and Narayan 2019). Strubbe and Quataert (2009) consider a simple model in which the radiation energy responsible for wind-launching is generated when returning stellar debris continuously hits the already formed disc at a distance close to $R_{c}=2 R_{p}$. A constant fraction $f_{\text {out }} \equiv \dot{M}_{\text {wind }} / \dot{M}_{\mathrm{fb}} \approx 0.1-0.3$ of the fallback debris is assumed to become a spherical wind, which is launched at $R_{c}$ with a velocity close to the escape velocity at $R_{c}$ and adiabatically expands. Inside $R_{c}$ a slim disc model similar to Abramowicz et al. (1988) is adopted to describe the disc properties. In particular, the disc temperature $T_{\mathrm{d}}$ is obtained as follows:

$$
\sigma_{\mathrm{SB}} T_{\mathrm{d}}^{4}=\frac{3 G M_{\mathrm{BH}} \dot{M} f}{8 \pi R^{3}}\left[\frac{1}{2}+\left(\frac{1}{4}+\left(\frac{\dot{M}}{\eta \dot{M}_{\mathrm{Edd}}}\right)^{2}\left(\frac{R_{\mathrm{s}}}{R}\right)^{2}\right)\right]^{-1},
$$

where $f=1-\sqrt{R_{\text {in }} / R}, R_{\mathrm{s}}$ is the Schwarzschild radius and the accretion rate in the disc is given by $\dot{M}=\left(1-f_{\text {out }}\right) \dot{M}_{\mathrm{fb}}$. In this model, strong optical emissions can be produced from the wind which cools down to few $\times 10^{4} \mathrm{~K}$ after adiabatic expansion. The total emission is dominated by wind emission at earlier time (for $\sim 10-100$ days depending on the exact stellar and black hole parameters and the wind launching radius) when the wind is optically thick. At later times, the wind becomes more dilute and the photosphere recedes, revealing more disc emissions. 


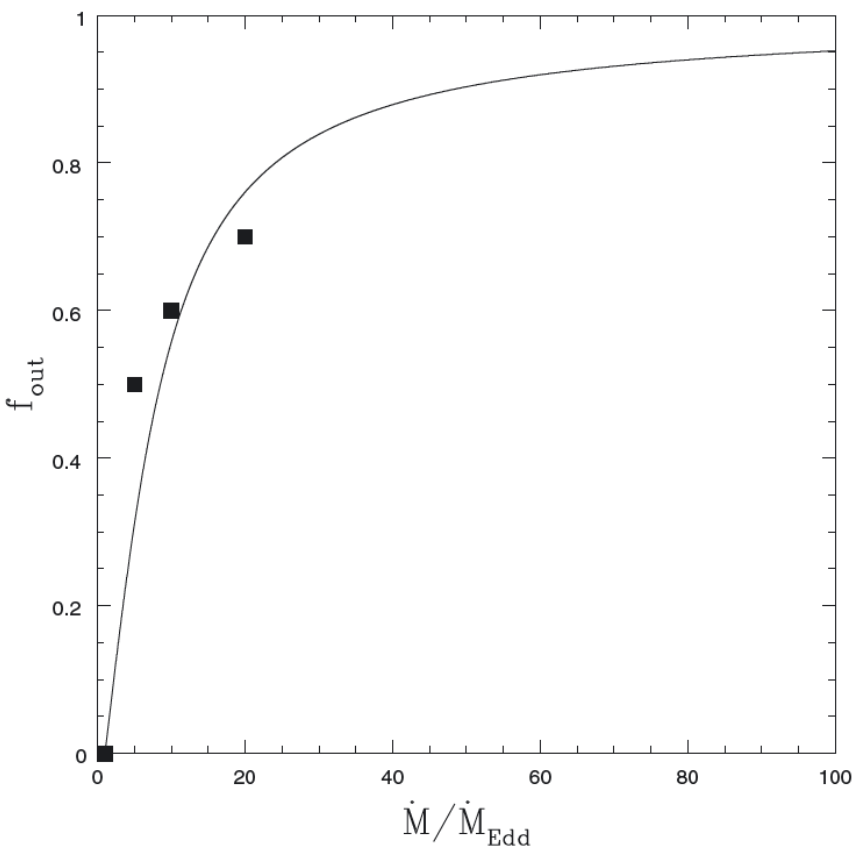

Fig. 5 The solid line shows the wind mass rate fraction $f_{\text {out }}$ as a function of the fallback rate of stellar debris in unit of the Eddington accretion rate, which is used by Lodato and Rossi (2011) to model the wind launched in TDE super-Eddington accretion discs. As the Eddington ratio increases, more fallback debris material becomes wind instead of accreting onto the black hole. The squares show the numerical results of Dotan and Shaviv (2011). Figure is from Lodato and Rossi (2011).

Lodato and Rossi (2011) consider a more realistic model for the wind produced from the radiation-pressure supported disc formed in the superEddington phase, based on the simulation of Dotan and Shaviv (2011). The wind mass loss fraction $f_{\text {out }}$ is taken to be larger at earlier times when the debris supply rate is higher (Fig. 5). They also calculate the emission produced by the slim, compact disc, assuming that the accretion rate through the disc is a constant fraction of the fallback rate. They find that, even if the disc bolometric luminosity scales as the fallback rate, as $t^{-5 / 3}$, the lightcurve in specific bands scales differently with time, because the disc temperature also evolves as the accretion rate declines. In particular, at wavelengths (such as in the UV and optical) that sit on the Rayleigh-Jeans tail of the disc multi-color blackbody, the lightcurve is proportional to the temperature and therefore scales as $T_{\mathrm{d}} \propto L_{\text {bol }}^{1 / 4} \propto t^{-5 / 12}$, declining much slower than the fallback rate. Conversely, for those wavelengths that are close to the peak of the blackbody at the beginning of the flare (i.e., in the soft X-rays, typically), the lightcurve initially declines as $t^{-5 / 3}$ (see Fig. 6). In general, the lightcurve is expected to evolve from a $t^{-5 / 12}$ decline, to a $t^{-5 / 3}$ and eventually to an exponential 


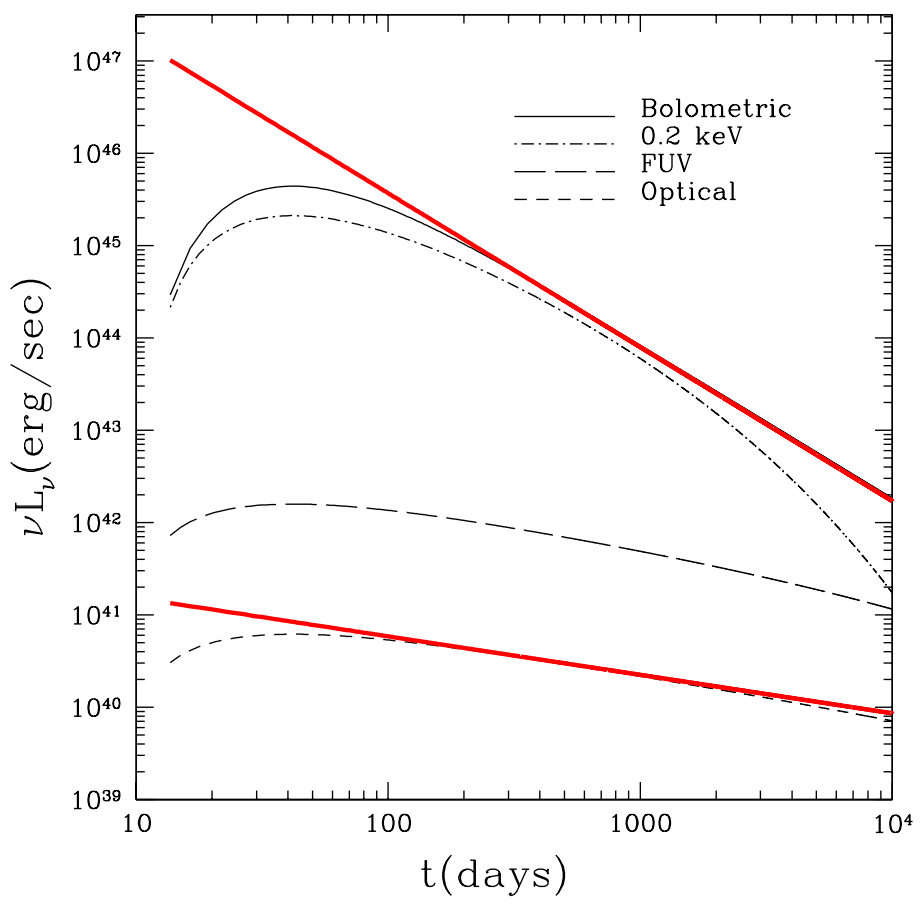

Fig. 6 Example lightcurves for the disc emission from the disruption of a solar type star by a $10^{6} M_{\odot}$ black hole at $\beta=1$. The fallback rate corresponds to a $\gamma=1.4$ polytropic model for the star (Lodato et al. 2009). The solid line shows the bolometric luminosity, the dot-dashed line shows the luminosity at $0.2 \mathrm{keV}$, the long-dashed line shows the luminosity in the FUV, at $\nu=1.910^{15} \mathrm{~Hz}$, while the short-dashed line shows the luminosity in the optical at $\nu=6.310^{14} \mathrm{~Hz}$. The two red lines mark the simple power laws expected for the bolometric luminosity $\left(\propto t^{-5 / 3}\right)$ and for the monochromatic luminosity in the optical/UV $\left(\propto t^{-5 / 12}\right)$. Figure is from Lodato and Rossi (2011).

decline with time. For high enough photon energies (e.g., in the X-rays), the first phase could be missing, and the ligthcurve would start directly from a close to $t^{-5 / 3}$ decline. If one also adds the wind emission in addition to the disc emission, the lightcurve gets further complicated and depends strongly on the system parameters, with the apparent lightcurve evolving coincidentially as $t^{-5 / 3}$ in particular regions of the parameter space.

These two models discussed previously assume that a sizable fraction of the fallback debris can make its way to the inner disc and super-Eddington accretion (onto the black hole) still happens. Instead, Miller (2015) and Metzger and Stone (2016) propose models in which most of the debris supply is launched as winds faraway from the black hole, either due to large opacities in the outer disc or due to shocks happening during the disc formation process. Under such scenarios, only a small fraction $(\lesssim 1 \%)$ of debris materials is accreted so the accretion rate is always sub-Eddington. The low level 
of accretion, together with the very mass-loaded wind functioning as a thick reprocessing envelope, can also reduce temperature of the TDE emission to few $\times 10^{4} \mathrm{~K}$ so that UV/optical emissions dominate.

Furthermore, numerical simulations of TDE discs with super-Eddington accretion rates (Dai et al. 2018; Curd and Narayan 2019) also show that strong winds can be launched in this phase, and we will discuss these numerical work in the next section.

\subsubsection{Simulated super-Eddington accretion flow and inclination dependence}

Realistically, we do not expect accretion flows to be spherically symmetric. While the studies mentioned in Section 2.1.1 and 2.1.2 mostly adopt 1D disc or envelope models when carrying out the semi-analytical calculations, they also suggest that the structure and emission of TDE accretion flow should have a dependence on the observer's viewing-angle with respect to the disc orientation. The details, however, can only be calculated using numerical simulations due to the complexity of the problem.

Codes that can be used to calculate the dynamics of super-Eddington accretion discs and capture its basic radiative transfer processes have only been developed in the recent decade (Ohsuga et al. 2005; Ohsuga and Mineshige 2011; Jiang et al. 2014; Sadowski et al. 2013; McKinney et al. 2014). (See the Simulation Methods Chapter of this book for details.) These novel simulations reveal that super-Eddington accretion discs always launch strong, opticallythick winds due to their large radiation pressure. The winds obscure the discs from a large solid angle but has an inclination-dependent profile. Close to the pole, wind is dilute and ultra-fast speed $(v \gtrsim 0.1 c)$. Close to the disc, wind is dense and moves slower. Analytical models of super-Eddington accretion discs, such as the slim disc model discussed previously, however, usually do no predict super-Eddington discs launch winds.

In order to check the viewing-angle dependence of TDE super-Eddington discs, Dai et al. (2018) have carried out a 3D general-relativistic radiation magneto-hydrodynamic (GRRMHD) simulation using a code called HARMRAD (McKinney et al. 2014, 2015). For the initial condition, they use a small, Keplerian, geometrically thick disc with mass and angular momentum about one tenth of the original stellar mass and angular momentum. The simulation has been evolved to achieve an inflow equilibrium of about $100 R_{g}$ (which is about ten times larger compared to previous super-Eddington disc simulations), in order to ensure resolving the photosphere from most of the inclination angles. The structure of the accretion disc and wind can be seen in Fig. 7. The accretion disc is geometrically and optically thick. The wind, launched by a combination of radiation and magnetic pressure, is also optically thick from most inclinations except from at angles close to the pole. A jet is also launched by the Blandford-Znajek mechanism due to the set up of the simulation with a large black hole spin and sufficient poloidal magnetic field threading the disc.

Reprocessing of the inner disc X-ray emissions happens in the wind and outer disc. At the same time, the wind also expands and cools down. There- 


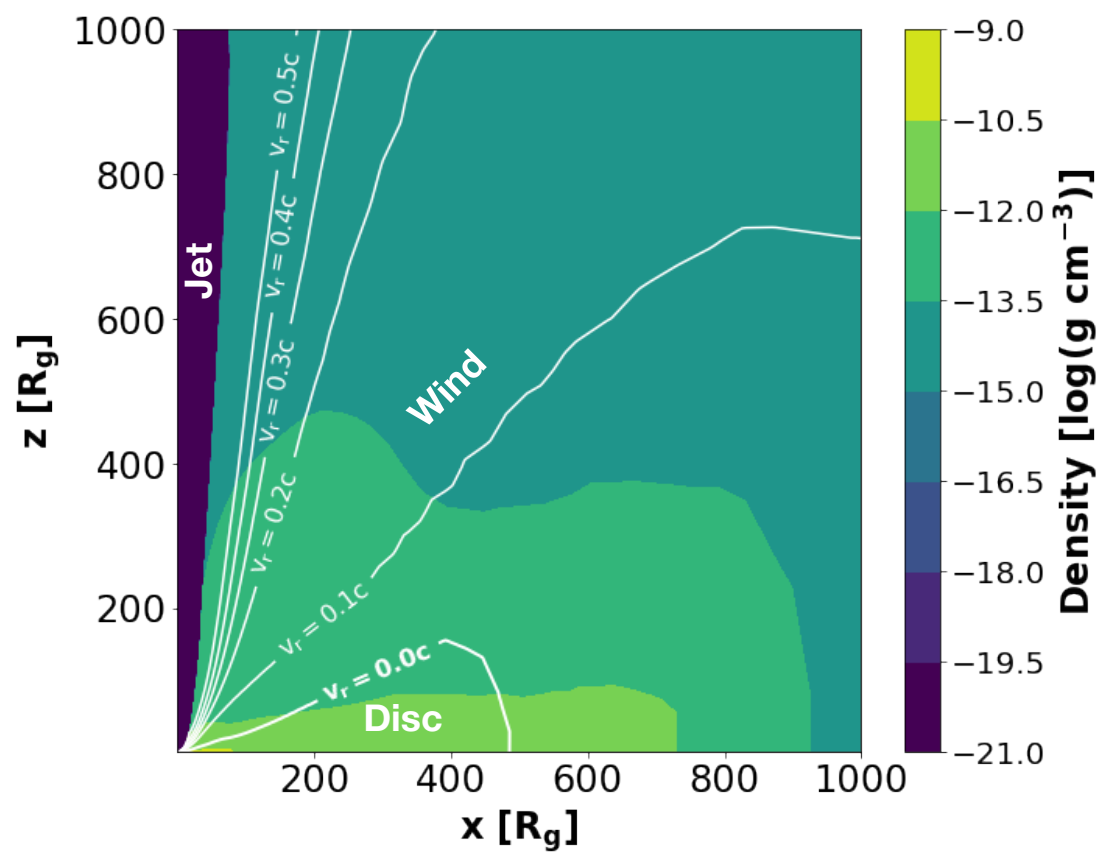

Fig. 7 Disc, wind and jet regions of the super-Eddington accretion flow simulated in Dai et al. (2018). The density of the disc and winds are shown by the background color. The white lines are contours of constant radial velocity in the wind region. Closer to the pole, the wind is more dilute and moves faster. The jet region (where magnetic pressure dominates the gas pressure) is marked using a dark blue colour. Figure is adapted from Thomsen et al. (2019).

fore, one should expect the wind emission to shine at lower energy bands due to the both adiabatic expansion and reprocessing as discussed in e.g., Strubbe and Quataert (2009); Roth et al. (2016); Metzger and Stone (2016). The simulated disc profile is then post-processed using a Monte-Carlo radiative transfer code SEDONA (Kasen et al. 2006; Roth et al. 2016) to obtain the spectra from different inclinations. It is found that UV/optical emission dominates when viewed along the disc direction, and X-ray emission dominates from directions closer to the pole. Interestingly, it is also found that most of the radiation energy will escape in the extreme ultraviolet (EUV) waveband which is heavily absorbed from extragalactic sources, and this might explain the "missing-energy" issue of the observed TDEs (Lu and Kumar 2018). Based on the results, Dai et al. (2018) propose a unified model for the observed UV/optical and X-ray TDEs based on the viewing-angle dependent structure of the accretion flow.

Curd and Narayan (2019) further simulate TDE super-Eddington discs with the GRRMHD code KORAL (Sadowski et al. 2013), using the ZEBRA 
model as disc initial conditions. While the first-order disc and wind structures are similar to those of Dai et al. (2018), since the initial large-scale structure of the accretion flow used in their simulations is more isotropic, the escaped emissions from different inclinations demonstrate less variance.

Both Dai et al. (2018) and Curd and Narayan (2019) have started the simulations with already circularized geometrically-thick small discs aligned with the black hole spin. Therefore, they cannot address the concerns related to the disc formation, such as whether TDE discs can indeed circularize promptly. To our knowledge, Sadowski et al. (2016) has carried out the only MHD simulation of TDEs from the disc formation phase to the accretion phase. Due to the constraint on computational time, the parameters chosen (i.e., an initial eccentric stellar orbit with a penetration parameter of 10) may only represent a small fraction of TDEs. Interestingly, it is found that while the disc can circularize fast, turbulence inherited from the violent debris stream self-interaction persists in the accretion flow, sustained either by convection or by the fall-back of gas. This hydrodynamic turbulence dominates over the magnetorotational instability (MRI) in the differentially rotating flow and contributes significantly to the angular momentum transfer.

\subsection{Late-time TDE disc models with disc spreading}

At late time in a TDE, as the accretion level gradually drops from superEddington to sub-Eddington regime, the disc thickness decreases and eventually should become a geometrically thin. The disc viscous or inflow timescale greatly increases with the reduced disc height. If the disc viscous timescale exceeds the debris fallback timescale, the draining rate of the disc through accretion will become lower than the mass supply rate of the returning debris, so material will accumulate in the disc.

Cannizzo et al. (1990) have calculated the evolution of a disc which initially has a ring structure with maximum surface density at $R \sim R_{t}$ and a total mass equal to a sizable fraction of the stellar mass. The disc is modeled using a standard $\alpha$-disc prescription with a viscosity parameter $\alpha=0.01-1$. As disc spreads both outwards and inwards due to gas pressure, its surface density decreases as shown in Fig. 8 and the disc temperate drops. While the exact accretion rate depends on the initial disc mass as well as the value of the disc viscosity and opacity, at later times the accretion rate adopts the same decline pattern with $\dot{M} \propto t^{-1.2}$, which is flatter than the canonical debris fallback rate. This model has been further revised by van Velzen et al. (2019) and Mummery and Balbus (2020) to explain the late-time light curves of TDEs observed in the UV or X-ray bands (e.g., Gezari et al. 2017; Holoien et al. 2018).

It has been a long-standing unsolved problem whether run-away thermal instability happens in radiation pressure-dominated thin accretion discs (Shakura and Sunyaev 1973). Shen and Matzner (2014) investigate this topic in the context of TDEs discs and predict that thermal instability, if it happens, 


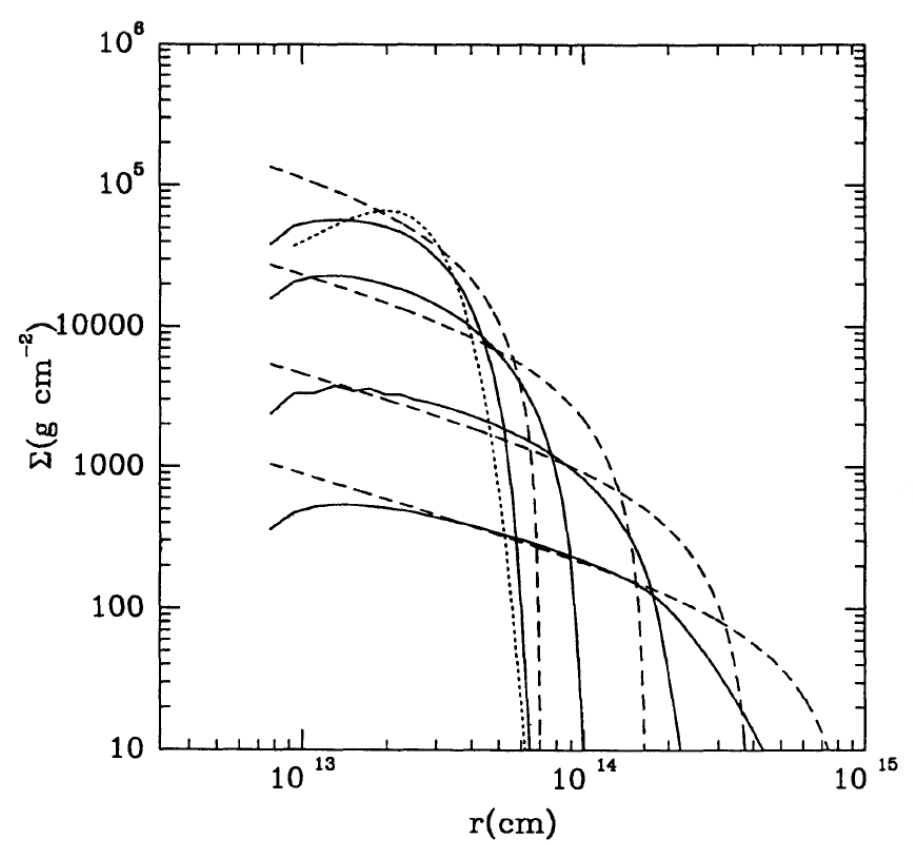

Fig. 8 Evolution of the surface density in the disc model by Cannizzo et al. (1990). The ring-structured disc has a mass of $0.5 M_{\odot}$ and viscosity parameter $\alpha=1$. The disc continues to spread out and surface density drops with the disc spreading. Curves show the surface density profile at the times of 0, 3, 30, 300, and 3000 yrs. Figure is from Cannizzo et al. (1990).

can lead to cycles of bursts of accretion. So far observed TDE flares have not demonstrated such behaviors. This perhaps suggests that thin discs formed in TDEs can stand against thermal instability either due to the support from high iron opacity (Jiang et al. 2016) or large-scale vertical magnetic field (Liska et al. 2019a), or due to the suppression of thermal instability from large-scale toroidal magnetic field (Begelman and Pringle 2007).

\subsection{Eccentric accretion flow formed in TDEs}

As current simulations show that nozzle shock happening at pericenter or debris stream self-crossing due to GR apisdal precession may not dissipate orbital energy fast enough, it is suggested that the just-formed disc can have an eccentric configuration. For example, for the stellar and black hole parameters in Shiokawa et al. (2015), the simulated accretion flow after several fall-back timescales is moderately eccentric. From the paper, Fig. 9 shows the time evolution of the radial profile of mass-weighted mean eccentricity, with the eccentricity settling to $e \sim 0.3-0.4$, extending from the inner-radius of the simulation $\left(\sim 30 R_{g}\right)$ to the minimum semi-major axis of the most bound debris 


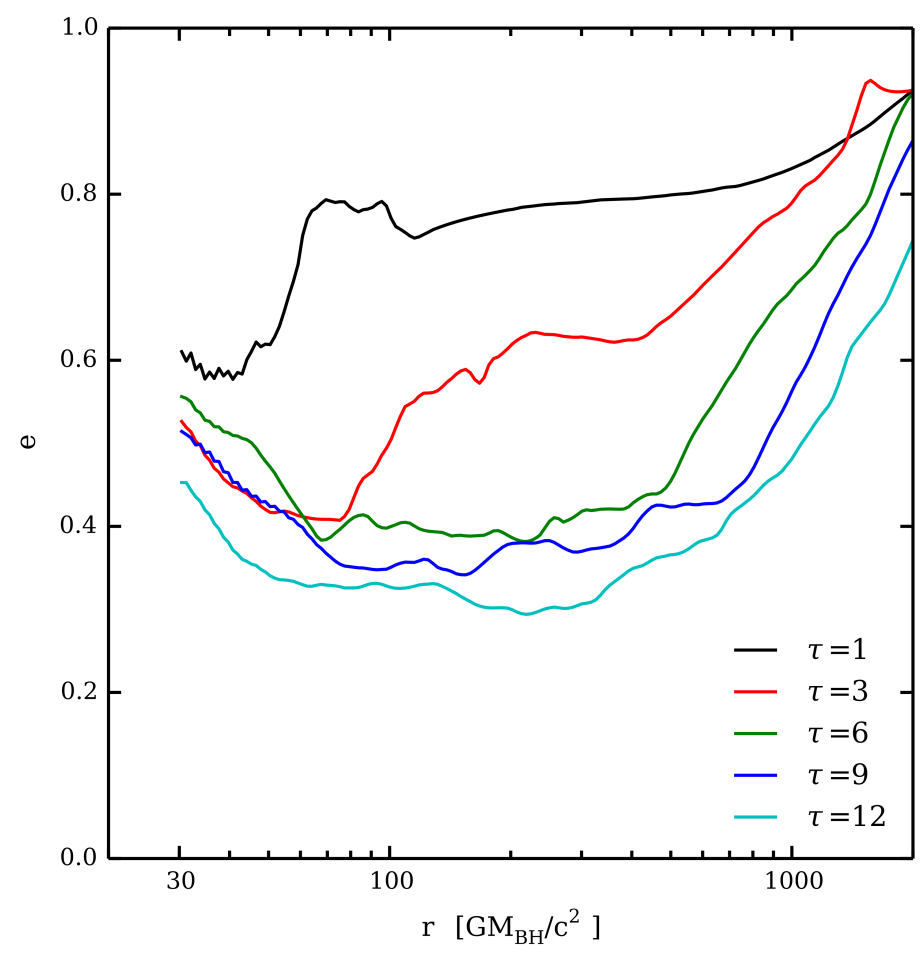

Fig. 9 Radial profile of eccentricity from the simulated accretion flow in Shiokawa et al. (2015). The time evolution of mass-weighted mean eccentricity is given in units of $\tau$, the orbital timescale of the most tightly bound matter. Note that the inner radius in this model is truncated at $30 R_{g}$. Figure is from Shiokawa et al. (2015).

$\left(\sim 500 R_{g}\right)$. Note that the tidal radius for this simulation is $\sim 100 R_{g}$, and the general relativistic hydrodynamics simulation does not include magnetic fields. These results, large disc and slow time scale for circularization, are contrary to the canonical model, where it is assumed that the accretion flow circularizes on the order of a few times the orbital timescale of the most tightly bound matter. The distinctive structure and time scale of the accretion flow imply that optical emission in TDEs could possibly be produced by mechanisms different from accretion onto the black hole.

It is also a topic under investigation whether eccentric accretion flow around black holes can generally circularize on timescale faster than the viscous timescale. In analytic treatments to the dynamics of eccentric discs, Syer and Clarke (1992) suggest that an initially eccentric accretion flow can maintain its configuration or even become more elliptical by applying Gauss perturbation equations upon a $\alpha$-viscosity disc model. Ogilvie and Barker (2014) and Barker and Ogilvie (2014) show that the eccentric disc evolution can depend on extreme, non-linear instabilities, providing a useful tool for understanding the leading mechanisms especially with follow-up numerical investigations. Based on this 
framework, Chan et al. (2018) show, with a toy model, how MRI growth occurs close to pericenter and declines with increasing eccentricity of the debris orbits. One remark is that the picture can become more complicated if one also considers general relativistic procession of the debris stream orbits and largescale magnetic fields in the disc, which is beyond the capacity of analytical treatments.

In light of this, several studies have turned towards emission produced during the evolution of an elliptical accretion flow. Such mechanisms are perhaps at the location of stream collision, at apocenter $a_{\text {min }}$ when the GR apsidal precession is weak (Piran et al. 2015a; Krolik et al. 2016). There are also many open questions in the evolution of eccentric accretion discs in the context of TDEs. For example, Svirski et al. (2017) address the issue of angular momentum loss by proposing a direct plunge of debris onto the black hole due to MHD stresses. We refer the readers to the Formation of the Accretion Flow Chapter in this book for more details.

Certain observational features of TDEs, especially double-peaked or flattopped broad $\mathrm{H} \alpha$ emission lines seen in a few candidates (e.g., Holoien et al. 2019), have motivated studies adopting thin elliptical disc models to explain the line profiles (Liu et al. 2017; Cao et al. 2018). For example, Eracleous et al. (1995) give expressions for the total line flux from the disc, which depend on a relativistic Doppler factor for photons in an elliptical structure, namely the eccentricity and inclination angle. Alternatively, such line profiles may also be modeled using circular discs with optically-thick winds (Roth and Kasen 2018; Hung et al. 2019).

\subsection{Angular momentum transport in TDE discs}

The issue of angular momentum transport in accretion discs has always been a significant source of controversy and investigations. For years, a phenomenological description based on a viscous disc model has been used, following the pioneering work of Shakura and Sunyaev (1973), until it was recognized that turbulence induced by the magneto-rotational instability (MRI) is the most likely origin for the required stress within the disc (see, e.g. Balbus and Hawley 1998 and references therein). Are discs originating from TDEs consistent with the established models for longer lived discs around Active Galactic Nuclei (AGN) and galactic black hole binaries? The question arises because the initial magnetic field of the star is expected to be relatively low, and the process of disruption itself is not expected to provide a significant enhancement in the magnetic field strength (Bonnerot et al. 2017; Guillochon and McCourt 2017).

Roughly speaking, one would imagine that, in order for the MRI to be established, the magnetic field should be strong enough that the Alfven speed is comparable to the gas sound speed. A full understanding of how MRI functions and how magnetic fields grow via MRI requires resolving the microphysical dissipation processes at small scales in the plasma. Global disc simulations have so far demonstrated that MRI can still kick in at significantly small magnetic 
field strengths, corresponding to a plasma $\beta$ value (the ratio of thermal to magnetic pressure) of the order of $\sim 100-1000$ (Bugli et al. 2018; Zhu and Stone 2018). If we apply this value for the conditions of typical of TDE discs, this would correspond to $B \sim 10^{3-4} \mathrm{G}$, much larger than the expected $1 \mathrm{G}$ field strength corresponding to the unmperturbed star. Recently, Nealon et al. (2018) have demonstrated how the torus resulting from a TDE can be unstable to the Papaloizou-Pringle instability (Papaloizou and Pringle 1984), that occurs for thick torii which are supported mainly by pressure against gravity. Such instability can provide a significant means of transport of angular momentum over the first $\sim 20$ orbits of the torus, before the MRI eventually develops, with the effective viscosity in the range of $\alpha \sim 0.03-0.05$. In addition, it could also provide a time-varying quadrupole moment that can give rise to potentially detectable gravitational wave emission (Toscani et al. 2019).

\section{Jets Launching in TDEs: How And When}

So far three TDEs have been observed to produce powerful jets: Swift J1644+57 (Bloom et al. 2011; Burrows et al. 2011; Zauderer et al. 2011), Swift J2058+05 (Cenko et al. 2012) and Swift J1112-82 (Brown et al. 2015). Their beamed hard $\mathrm{X}$-rays/soft $\gamma$-rays and the accompanied strong radio emissions have been interpreted to originate from relativistic, collimated jets formed in TDEs. This gives the most direct evidence that transient accretion discs form in TDEs. As we have discussed in Section 2, TDE discs transition from the super-Eddington accretion regime to the sub-Eddington thin disc regime and then eventually to the ADAF/RIAF regime, in a timescale much faster than typical AGN statechanging timescales. Therefore, TDEs can also be used to study jet physics in all three accretion regimes. In this section, we mainly focus on the jetlaunching physics, and leave the jet emission physics to be discussed in details in the Emission Mechanisms Chapter, X-ray Chapter and Radio Chapter.

The best studied jet launching mechanism is the Blandford-Znajek (hereafter BZ) mechanism, in which relativistic jet can be powered by extracting the $\mathrm{BH}$ rotation energy via ordered magnetic field threading the ergosphere of a spinning black hole (Blandford and Znajek 1977). In the standard BZ approximation, the $\mathrm{BH}$ energy extraction rate scales as $a^{2} \Phi_{B}^{2}$ ( $a$ is the black hole spin parameter and $\Phi_{B}$ is the large-scale magnetic flux around the black hole). This has been demonstrated in GRMHD simulations (e.g., McKinney 2005; Tchekhovskoy et al. 2011; McKinney et al. 2012). Under certain conditions the jet power can increase even faster than $a^{2}$ for large valules of $a$ (Tchekhovskoy et al. 2012).

The accretion flow around an astrophysical BH plays a crucial role in transporting magnetic flux to its vicinity, amplifying magnetic flux via MRI or dynamo process, and confining magnetic flux from leaking (e.g., Pessah and Psaltis 2005; Beckwith et al. 2009). Since it is easier for magnetic flux to accumulate in geometrically thick discs (Lubow et al. 1994), powerful, continuous jets are expected to be launched in the super-Eddington regime or 


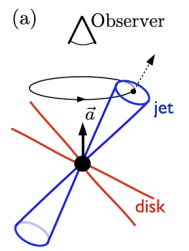

\section{Stage 1}

Precessing

$\sim$ few weeks

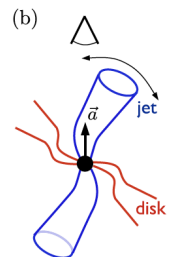

Stage 2

Wobbling jet

$\sim$ few weeks (c)

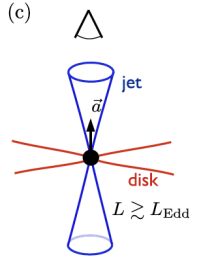

Stage 3

Steady

spin-aligned jet

$\sim 1.5$ years (d)

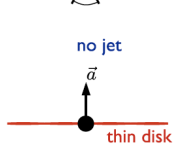

(e) $\triangle$

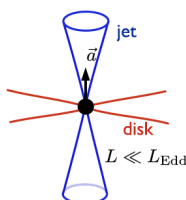

Stage 5

Jet revival

Unlimited

Fig. 10 Evolution of the jet and disc in Swift J1644 proposed by Tchekhovskoy et al. (2014). A misaligned jet initially forms in the super-Eddington accretion phase, which precesses and wobbles around the black hole spin axis. Within weeks the jet orientation is aligned with black hole spin axis due to the magneto-spin alignment mechanism. After about one year, the disc becomes sub-Eddington, so the jet is quenched. As the accretion accretion continues to drop, the jet is predicted to revive when the disc eventually transitions to the ADAF/RIAF accretion regime. Figure is from Tchekhovskoy et al. (2014).

the ADAF/RIAF regime. Furthermore, the thick disc geometry can help jet collimation.

The change of accretion regime has been used to explain the the X-ray light curve of the Swift J1644 event (Zauderer et al. 2013; Tchekhovskoy et al. 2014): Initially when the debris disc accretion rate was super-Eddington and the disc was geometrically thick, a relativistic, collimated jet was launched, producing strong X-ray and radio emissions. During this phase the jet power and X-ray flux roughly traced the debris fallback rate following the $t^{-5 / 3}$ pattern since about 10 days after the trigger. Then the $\mathrm{X}$-ray emission rapidly decreased by two orders of magnitude after about 500 days, by which time the fallback rate was expected to drop to around or below the Eddington level. As a consequence, the disc morphology changed and the jet quenched. This evolution, together with the initial disc-jet alignment process which will be discussed in Section 4, is illustrated in Fig. 10.

When are the most powerful jets produced? For any accretion disc there exists an upper limit of the magnetic flux that the disc can confine near the black hole. When the magnetic flux exceeds this limit, the magnetic pressure becomes stronger than the disc gravity and pushes the accretion flow away from the black hole. The type of accretion flow under the strong magnetic-field limit is called the magnetically-arrested disc (MAD) (Narayan et al. 2003). One can define a dimensionless magnetic flux parameter:

$$
\Upsilon \equiv \frac{0.7 \Phi}{\sqrt{4 \pi R_{g}^{2} \dot{M} c^{2}}},
$$

where $\Phi$ is the flux of poloidal magnetic flux threading the disc in Gaussian units and $\dot{M}$ is the accretion rate. The disc is in the MAD state when $\Upsilon \gg 1$. 
GRMHD simulations show that the most powerful jets with large Lorentz factor are launched from MAD discs, which can achieve a jet efficiency $\eta_{\text {jet }} \equiv$ $L_{\text {jet }} / \dot{M}_{\mathrm{BH}} c^{2} \approx 10-100 \%$ (Hawley and Krolik 2006; Tchekhovskoy et al. 2011, 2012; McKinney et al. 2012). On the other hand, weakly magnetized discs in the standard and normal evolution (SANE) regime (Narayan et al. 2012) can only produce low-power jets or non-relativistic outflows in the polar region (De Villiers et al. 2005; Mościbrodzka et al. 2016). Recent GR-radiationMHD simulations of super-Eddington (TDE) discs also show consistent results (McKinney et al. 2015; Sadowski and Narayan 2016; Dai et al. 2018; Curd and Narayan 2019).

If the Swift J1644 jet is launched from a MAD disc by the BZ mechanism, then the magnetic flux threading the $\mathrm{BH}$ needed to explain the peak X-ray luminosity is about $10^{30} \mathrm{G} \mathrm{cm}^{2} \times\left(M_{\mathrm{BH}} / 10^{6} M_{\odot}\right)$ (Bloom et al. 2011; Kelley et al. 2014), which is $3-5$ orders of magnitude larger than the original magnetic flux possessed by a main-sequence star. This therefore has triggered a series of investigations on how magnetic flux can be amplified during the disruption, disc forming or accretion processes in TDEs. Simulations studying the magnetic field amplification during the disruption process (Guillochon and McCourt 2017; Bonnerot et al. 2017) show that although the dynamo process in surviving cores from partial disruption events might be important, the amplification in returning debris in a total disruption event is not significant enough to supply the flux needed for the Swift J1644 jet. From the prospect of magnetic-field growth via MRI in accretion discs, Krolik and Piran (2011) propose that Swift J1644 might have been produced by an intermediate mass black hole (IMBH) disrupting a white dwarf. In this scenario the disc orbital timescale is shorter than the case of a SMBH disrupting a main-sequence star, so the magnetic field can quickly grow via turbulence to reach saturation. (However, Kara et al. (2016) later constrains the SMBH mass in Swift J1644 to be a few $\times 10^{6} M_{\odot}$ from X-ray reverberation studies, which favors the disruption of a main-sequence star instead.) Kelley et al. (2014) propose another model in which extra magnetic fields can be collected by the returning debris when it sweeps through a "fossil" disc left from previous AGN activities. When the stellar orbit and the fossil disc aligns, the magnetic flux that a quiescent fossil disc can provide to the debris is enough to power the Swift J1644 jet.

Alternatively, it has been suggested that in the TDE super-Eddington accretion phase a jet can be driven by the large radiation pressure and collimated by the gemetrically thick accretion flow (Coughlin and Begelman 2014; Sadowski and Narayan 2015). This jet production mechanism, however, has not been studied in details. More studies on this topic are needed to conclude whether relativistic jets can be produced purely due to radiation pressure.

\section{Precession And Alignment of TDE Discs And Jets}

One peculiar feature of TDEs is that in general we expect the orbit of the incoming star (and thus the angular momentum of the debris) to be randomly 
oriented with respect to the spin of the central supermassive black hole, with the latter determined by the merger history of the host galaxy and the accretion history of the black hole. Since the stellar pericenter is relatively close to the gravitational radius of the hole, we expect the debris to be significantly affected by relativistic effects in the form of Lense-Thirring precession. Such effects might delay the formation of a disc, by avoiding stream collisions due to a randomization of the orbital plane (Dai et al. 2013; Guillochon and Ramirez-Ruiz 2015; Hayasaki et al. 2016), or might speed-up circularization, by producing a thicker torus (Gafton and Rosswog 2019). Either way, after the disc is formed, it is expected that the initial orientation of the disc will be misaligned with the black hole spin due to conservation of angular momentum. Also, misaligned discs may also launch jets, and the jet orientation can be affected by both the disc angular momentum and the black hole angular momentum. In general, since such misalignment are specific to TDEs and might strongly affect the evolution of the system, several studies have investigated the dynamics of misaligned discs and jets in TDEs (Stone and Loeb 2012; Lei et al. 2013; Tchekhovskoy et al. 2014; Franchini et al. 2016; Xiang-Gruess et al. 2016; Ivanov et al. 2018; Zanazzi and Lai 2019).

\subsection{Expected precession regimes in TDE discs}

The main physical effect at work in misaligned discs around a spinning black hole is Lense-Thirring precession. The precession torque at radius $R$ is given by (see, for example, Franchini et al. 2016 and references therein):

$$
\mathbf{T}=-\Sigma R^{2} \Omega\left(\frac{\Omega_{z}^{2}-\Omega^{2}}{\Omega^{2}}\right) \frac{\Omega}{2} \mathbf{e}_{\mathbf{z}} \times \mathbf{1},
$$

where $\Sigma$ is the disc surface density, $\mathbf{l}$ is the unit vector along the local disc angular momentum direction, $\mathbf{e}_{z}$ is the direction of the black hole spin, and $\Omega$ and $\Omega_{z}$ are the angular and vertical frequencies, respectively, given by:

$$
\begin{gathered}
\Omega=\frac{c^{3}}{G M} \frac{1}{r^{3 / 2}+a} ; \\
\left(\frac{\Omega^{2}-\Omega_{z}^{2}}{\Omega^{2}}\right)=4 a r^{-3 / 2}-3 a^{2} r^{-2},
\end{gathered}
$$

where $M$ is the black hole mass, $r$ is the disc radius in units of the gravitational radius of the hole, and $a$ is the black hole spin parameter.

The response of the disc to external misaligned torques has been studied extensively over the years. The pioneering work of Papaloizou and Pringle (1983) and of Papaloizou and Lin (1995) have shown that the disc responds to such perturbations either in a diffusive way (when the ratio of turbulent stresses to pressure $\alpha$ is larger than the disc aspect ratio $H / R$ ) or in a wavelike way (in the opposite regime, when $\alpha \lesssim H / R$ ). A full non-linear theory of diffusive warp propagation has been provided by Ogilvie (1999). On the 
other hand, a non-linear theory of bending waves has not been developed, and only a linear (Lubow and Ogilvie 2000) and quasi-linear theory (Ogilvie 2006) exist. Non-linearities might prevent the wave-like regime from happening altogether (Hawley and Krolik 2018). For thin and viscous discs, or for discs that evolve diffusively, the inner disc aligns rapidly to the black hole spin (on the warp diffusion timescale), while the outer disc retains its original direction and slowly aligns at longer times. This is known as the Bardeen-Petterson effects (Bardeen and Petterson 1975).

In the case of TDE discs, we expect initially the disc to be thick (large $H / R$ ) due to the high accretion level and with a relatively low value of internal "viscous" stresses, as the magneto-rotational instability is building up (low $\alpha$ ), and should therefore be characterized by bending wave propagation. At later times, however, the disc becomes thinner and magnetic stresses build up, so a transition to the diffusive regime is expected to occur.

4.2 Global disc and jet precession in the thick disc regime

At early times in TDEs, regular global precession of the disc might occur, as shown in the GRMHD simulations of thick torii (Fragile and Anninos 2005; Fragile et al. 2007; Liska et al. 2018). For TDEs, this was initially suggested by Stone and Loeb (2012) and later confirmed with time-dependent calculations by Franchini et al. (2016). The global precessional frequency can be computed easily as a weighted average of the Lense-Thirring frequency $\Omega_{\mathrm{LT}}$ (Stone and Loeb 2012; Franchini et al. 2016):

$$
\Omega_{\mathrm{p}}=\frac{\int_{R_{\text {in }}}^{R_{\text {out }}} \Omega_{\mathrm{LT}}(R) L(R) 2 \pi R d R}{\int_{R_{\text {in }}}^{R_{\text {out }}} L(R) 2 \pi R d R},
$$

where $L(R)=\Omega R^{2} \Sigma$. Equation (9) is applicable when the disc is narrow (as expected for TDE) and the warp is mild (Note that a mild warp does not imply a mild misalignment). The Lense-Thirring frequency is easily derived from Eqs. (6) and (8):

$$
\Omega_{\mathrm{LT}}(r)=\frac{1}{2} \frac{c^{3}}{G M} \frac{1}{r^{3 / 2}+a}\left(4 a r^{-3 / 2}-3 a^{2} r^{-2}\right) .
$$

The resulting precession periods are in the range of a few to several days, depending on black hole mass and spin, and become longer for lower spins and lower black hole masses (see Fig. 11, taken from Franchini et al. 2016). However, it should be noted that such periods strongly depend on where the disc lies, given the steep scaling of the Lense-Thirring rate with radius. If, for some reason, the disc lies much closer to the hole, the precession rate could be much higher. This will also occur if the disc is torn into smaller rings, in cases where the misalignment is very large (Nixon et al. 2012).

A few recent studies have explored the effect of the torques induced by infalling material from the debris stream onto the disc. It is found that the 


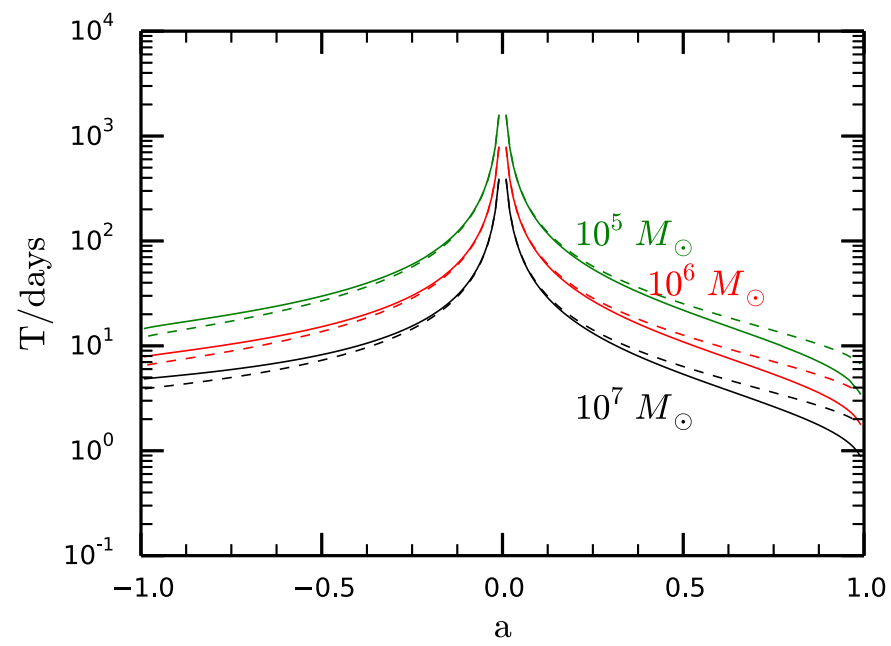

Fig. 11 Precession period expected from a TDE disc for three different choices of SMBH mass (green: $10^{5} M_{\odot}$, red: $10^{6} M_{\odot}$, black: $10^{7} M_{\odot}$. Solid line refer to the full relativistic calculation, while dashed line adopt a first order approximation to the Lense-Thirring frequency. These model refer to disc that extend out to $2 R_{\mathrm{t}}$, for $\beta=1$. Figure is from Franchini et al. (2016)

effect of such torques is to complicate the disc dynamics, leading to irregular precessional motion of the disc (Xiang-Gruess et al. 2016; Ivanov et al. 2018), possibly giving rise to quasi-periodic, rather than period precession.

Recent GRMHD simulations have shown that tilted discs can also launch relativistic jets (McKinney et al. 2013; Liska et al. 2018). When the disc is not highly magnetized, the jet has been shown to initially follow the disc angular momentum orientation on large scales and precess together with the disc, although the tilt angle between the black hole spin and the disc/jet decreases over longer timescales. (See section 4.4 for the alternative case of highly magnetized discs.)

\subsection{Viscous vs cooling driven disc alignment}

Over long timescales, independently on the original misalignment, the disc is expected to settle down on the equatorial plane of the black hole due to dissipative effects (Sorathia et al. 2013b,a), although some non dissipative relaxation processes have been sometimes suggested for stellar systems (Merritt and Vasiliev 2012), the applicability of which to TDE discs has not been shown yet. Stone and Loeb (2012) argue that this would have naturally happened due to the fact that, as the accretion rate through the disc decreases (for example, following the fallback rate, as $t^{-5 / 3}$ ), the disc would become thinner and grad- 
ually move to the Bardeen-Petterson regime, at which stage rapid alignment would follow. However, even in the wave-like propagation phase, alignment is expected to happen as a consequence of viscous dissipation. Franchini et al. (2016) evaluate specifically the relevant alignment process in the presence of both cooling driven disc thinning and of viscous effects in the disc. The two possible alignment processes have a distinctly different dependence on the viscosity parameter $\alpha$, whereby cooling induced alignment scales as $\alpha^{-3 / 5}$, while viscous induced alignment scales as $\alpha^{-1}$. Franchini et al. (2016) explore a wide range of parameters and conclude that for most cases, viscously induced alignment occurs more quickly that cooling induced alignment. The only cases for which the disc aligns due to the Bardeen-Petterson effect are for large black hole mass, low spins and low values of $\alpha$.

The alignment time is of the order of tens to hundreds of days for large black hole spin and grow to a few years for very low spins.

4.4 Magneto-spin alignment of strongly magnetized discs and jets

When large-scale electromagnetic (EM) field exists around the black hole, EM force can also affect the dynamics of jets and discs and provides another torque to affect their orientations. In the case of a highly magnetized disc, the strong coupling between the black hole spin and its magnetosphere can make not only the relativistic jet but also the inner part of a thick accretion disc align with the black hole spin axis.

One can compare the EM force torque and the torque induced by the LenseThirring effect to see which one dominates the disc dynamics. With simple assumptions such as that the magnetic field, when being pushed against by a disc, bends on the same scale of the disc radius $r$, McKinney et al. (2013) calculate that:

$$
\frac{\tau_{\mathrm{EM}}}{\tau_{L T}} \approx \frac{1}{64 a} \Upsilon^{2} \frac{r}{R_{g}} \alpha_{\mathrm{eff}}\left(\frac{H}{R}\right)^{2} .
$$

Here $a$ is the dimensionless black hole spin parameter, $\Upsilon$ is the dimensionless magnetic flux given by Eqn. $5, H / R$ is the disc aspect ratio, and $\alpha_{\text {eff }} \equiv$ $v_{r} /\left(v_{\phi}(H / R)^{2}\right)$ is the effective viscosity parameter. Therefore, the EM torque becomes more dominant when the disc is more magnetized, or when the disc is thicker. 3D GRMHD simulations and calculations (McKinney et al. 2013; Polko and McKinney 2017; Liska et al. 2018) have further demonstrated that for the highly magnetized thick discs (such as MAD discs) with a large range of tilt angles, the EM torque is dominant and strong enough to reorient the inner disc and inner jet to align with the $\mathrm{BH}$ spin axis.

Tidal disruption events likely produce thick accretion discs due to the high accretion level. These discs might also be strongly magnetized, especially when relativistic jets are produced which require large-scale magnetic fluxes to power (see Section 3). Therefore, EM force could play an important role in aligning the discs and jets with black hole spins in TDEs such as Swift J1644. 
4.5 Warping and tearing of thin accretion discs

In this regime which is typically assumed to happen at late times in TDEs, we expect the inner part of a thin disc not to precess regularly but to simply align with the black hole spin axis due to the Bardeen-Petterson effect. This has been demonstrated in Smoothed Particle Hydrodynamics simulations by Nelson and Papaloizou (2000); Lodato and Pringle (2007); Lodato and Price (2010) as well as in GRMHD simulations by Liska et al. (2019a,b). As the disc is fed by debris on fallback timescales, the outer part of the disc may not have sufficient time to be aligned and its orientation could be still determined by the angular momentum of the falling back debris. The disc can become warped or even torn apart (Nixon et al. 2012; Liska et al. 2019b). Zanazzi and Lai (2019) compute eigenmodes for the disc warp excited by Lense-Thirring precession and apply it to disc produced in TDEs. They compute global precession frequencies finding a broad agreement with those predicted by Franchini et al. (2016), with precession periods ranging from days to weeks depending on the disc parameters.

4.6 Possible cases of misaligned discs and jets observed in TDEs

\subsubsection{QPO in ASASSN 14-li}

From the observational side, ASASSN14-li interestingly shows a quasi-periodic oscillation in the soft x-ray band $(0.3-1 \mathrm{keV})$ with a periodicity of only 131 seconds with $3-4 \sigma$ significance (Pasham et al. 2019). It has been argued that this QPO is possibly associated with the global Lense-Thirring precession of a misaligned disc, though quantitative calculations pose several challenges. From Eqn.[10] such a high precession rate would require an extremely high spin for the black hole even if using the LT precession rate at the black hole ISCO radius. This case is further puzzling since the signal has been observed to be stable for roughly 450 days, which is much longer than the expected alignment process for such a high spin.

\subsubsection{Behavior of the relativistic jet in Swift J1644}

The X-ray level of Swift J1644 shows large-amplitude variability in the first few weeks (Bloom et al. 2011), which has been interpreted to be the result of jet wobbling (Tchekhovskoy et al. 2014). Then the jet and the highly magnetized disc have been aligned by the magneto-spin alignment mechanism as discussed in Section 4.4, as shown in Fig. 10. This model is further supported by the lack of clear evidence of jet precession during the main light curve decay phase lasting for about one year, although there is a report of $\mathrm{a} \sim 4 \sigma$ detection of a 200 second QPO in the $2-10 \mathrm{keV}$ X-ray band with an unknown nature (Reis et al. 2012). 


\section{Summary And Future Studies}

The studies of accretion discs, winds and jets in TDEs discussed in this review add an important component to the studies of general accretion and jet physics around black holes. The extreme level of debris accretion and the fast transition across different accretion regimes offer us unique opportunities of studying physics that do not in happen other accretion phenomena such as AGNs or $\mathrm{X}$-ray binaries.

The masses and spins of the SMBHs contain valuable information about their growth as well as the assembling and merger history of the galaxies since the early universe. TDEs are important probes to the demographics of SMBHs, since they can illuminate dormant $\mathrm{SMBHs}$, which represent the majority of the SMBH population at low redshifts, with bright flares lasting for years. Also, while AGN observations tend to select luminous, massive targets, TDEs allow us to study less massive galaxies and their SMBHs, since stars approaching black holes with $M \gtrsim 10^{8} M_{\odot}$ will typically plunge into the event horizon before being tidally disrupted (unless the SMBH is spinning very rapidly, see Kesden 2012).

A good understanding of the accretion and jet physics in TDEs will help us independently constrain SMBH parameters from TDE observables. For example, the power of a magnetically driven jet strongly correlates with the value of the black hole spin. Also, while the debris mass fallback rate has a

dependence on the SMBH mass (e.g., $\dot{M}_{\mathrm{fb} \text {,peak }} \propto M_{\mathrm{BH}}^{-1 / 2}$, as in Guillochon and Ramirez-Ruiz 2013) and therefore the TDE light curve can in principle be used to constrain the SMBH mass (Mockler et al. 2019), the luminosity (in monochromatic bands) may not exactly scale with the fallback rate, since the overall radiative effciency or the SED of the emission can change with accretion rate (Lodato and Rossi 2011; Piran et al. 2015b), letting alone the uncertainties and delays related to the disc formation process.

As of now most of the studies of TDE accretion flow have focused on the ideal case of circular and aligned discs. They have shown that winds and relativistic jets can be launched at early times when the accretion is in the super-Eddington regime and the disc geometry is thick. These studies also offer explanations for the basic emission properties of TDEs, such as how X-ray emission produced close to the black hole can be reprocessed into UV/optical emissions in optically-thick winds or outer accretion flow. Studies of TDE discs at late times in the sub-Eddignton phase show that such unobscured thin discs should mostly produce X-ray emissions. They have long viscous timescales so they can keep accumulating mass and significantly spread outwards on debris fallback timescales. Recently, more explorations have been made into less understood regimes such as misaligned discs/jets or eccentric accretion discs. A lot of the progress as discussed in this chapter has been made possible largely thanks to various novel numerical simulations carried out recently to investigate accretion flow and jets which are too complex to be studied analytically.

At the end of this review, we pose a few open questions to motivate future studies: 
* Recently more TDEs have been followed up for several years past the peak of the flare. This offers a good opportunity for further studying thin accretion discs and especially the transition between the super-Eddington regime and the thin disc regime. For example, do TDE emissions at late times share similar properties as AGN emissions which are assumed to be produced from thin accretion discs?

* If, in a TDE, the disc is initially highly eccentric, how does such a disc evolve? Can it maintain an eccentric configuration for a long time or circularize quickly? How does MRI work in such discs? Can eccentric discs also generate winds and jets?

* The basic emission properties of TDEs and some of their spectroscopic features can be qualitatively explained using current accretion and emission models. For example, from the observed Bowen fluorescent lines in several optically strong TDEs, we know from first order calculations that there exists an optically-thick reprocessing layer that converts X-ray/EUV emissions into optical emissions (Leloudas et al. 2019). Also the asymmetric, broad emission line profiles in some TDEs (Hung et al. 2019; Holoien et al. 2019) indicate that these discs either produce winds or have an eccentric geometry. In the future more quantitative studies along these lines will be very useful for studying the properties of the accretion flow.

* How can we apply TDE accretion and jet physics to study other accretion phenomena, such as AGNs, Galactic X-ray binaries, ultra-luminous X-ray sources and gamma-ray bursts? And vice versa?

* The emission from a typical X-ray strong AGN always has a power-law hard $\mathrm{X}$-ray component, which is believed to be produced by the Compotonization of the cold disc photons by a hot "corona" above the disc. However, (non-jetted) X-ray strong TDEs only produce soft thermal emissions. What makes coronae disappear in TDE discs?

* If most TDE discs are surrounded by large, optically-thick reprocessing layers (in the form of steady envelopes or winds), then it may not be straightforward to directly probe the space-time very close to black holes with TDE observables. Perhaps X-ray strong TDEs and jetted TDEs give us the best hope for constraining black hole spins.

In the future, detailed models and simulations of disc formation, accretion and emission processes will help us better answer these questions in our quest of probing the SMBH demographics, black hole accretion/jet physics and general relativity with potential samples of thousands of tidal disruption events.

Acknowledgements The authors thank the International Space Science Institute (ISSI) for their support and hospitality and the review organisers for their leadership in coordinating these reviews. The authors also thank the Yukawa Institute for Theoretical Physics (YITP) at Kyoto University for hosting the International Molecule-type Workshop "Tidal Disruption Events: General Relativistic Transients" (YITP-T-19-07), where the authors held useful discussions to complete this work. The authors are grateful to the referees for providing constructive suggestions and comments. JLD is supported by the GRF grant from the Hong Kong government under HKU 27305119. The work of RMC is funded by the Advanced Simulation Computing Physics and Engineering Program under the auspices of Los Alamos 
National Laboratory, operated by Triad National Security, LLC, for the National Nuclear Security Administration of U.S. Department of Energy (Contract No. 89233218CNA000001).

\section{References}

M.A. Abramowicz, B. Czerny, J.P. Lasota, E. Szuszkiewicz, Slim accretion disks. Astrophys. J. 332, 646-658 (1988). doi:10.1086/166683

K.D. Alexander, E. Berger, J. Guillochon, B.A. Zauderer, P.K.G. Williams, Discovery of an Outflow from Radio Observations of the Tidal Disruption Event ASASSN-14li. Astrophys. J. Lett. 819, 25 (2016). doi:10.3847/2041-8205/819/2/L25

I. Arcavi, A. Gal-Yam, M. Sullivan, Y.-C. Pan, S.B. Cenko, A. Horesh, E.O. Ofek, A. De Cia, L. Yan, C.-W. Yang, D.A. Howell, D. Tal, S.R. Kulkarni, S.P. Tendulkar, S. Tang, D. Xu, A. Sternberg, J.G. Cohen, J.S. Bloom, P.E. Nugent, M.M. Kasliwal, D.A. Perley, R.M. Quimby, A.A. Miller, C.A. Theissen, R.R. Laher, A Continuum of H- to He-rich Tidal Disruption Candidates With a Preference for E+A Galaxies. Astrophys. J. 793, 38 (2014). doi:10.1088/0004-637X/793/1/38

K. Auchettl, J. Guillochon, E. Ramirez-Ruiz, New Physical Insights about Tidal Disruption Events from a Comprehensive Observational Inventory at X-Ray Wavelengths. Astrophys. J. 838(2), 149 (2017). doi:10.3847/1538-4357/aa633b

S.A. Balbus, J.F. Hawley, Instability, turbulence, and enhanced transport in accretion disks. Reviews of Modern Physics 70, 1-53 (1998). doi:10.1103/RevModPhys.70.1

J.M. Bardeen, J.A. Petterson, The Lense-Thirring Effect and Accretion Disks around Kerr Black Holes. Astrophys. J. Lett. 195, 65 (1975). doi:10.1086/181711

A.J. Barker, G.I. Ogilvie, Hydrodynamic instability in eccentric astrophysical discs. Mon. Not. R. Astron. Soc. 445(3), 2637-2654 (2014). doi:10.1093/mnras/stu1939

K. Beckwith, J.F. Hawley, J.H. Krolik, Transport of Large-Scale Poloidal Flux in Black Hole Accretion. Astrophys. J. 707(1), 428-445 (2009). doi:10.1088/0004-637X/707/1/428

M.C. Begelman, J.E. Pringle, Accretion discs with strong toroidal magnetic fields. Mon. Not. R. Astron. Soc. 375(3), 1070-1076 (2007). doi:10.1111/j.1365-2966.2006.11372.x

R.D. Blandford, D.G. Payne, Hydromagnetic flows from accretion disks and the production of radio jets. Mon. Not. R. Astron. Soc. 199, 883-903 (1982). doi:10.1093/mnras/199.4.883

R.D. Blandford, R.L. Znajek, Electromagnetic extraction of energy from Kerr black holes. Mon. Not. R. Astron. Soc. 179, 433-456 (1977). doi:10.1093/mnras/179.3.433

R.D. Blandford, M.C. Begelman, On the fate of gas accreting at a low rate on to a black hole. Mon. Not. R. Astron. Soc. 303(1), 1-5 (1999). doi:10.1046/j.1365-8711.1999.02358.x

R.D. Blandford, M.C. Begelman, Two-dimensional adiabatic flows on to a black hole - I. Fluid accretion. Mon. Not. R. Astron. Soc. 349(1), 68-86 (2004). doi:10.1111/j.13652966.2004.07425.x

J.S. Bloom, D. Giannios, B.D. Metzger, S.B. Cenko, D.A. Perley, N.R. Butler, N.R. Tanvir, A.J. Levan, P.T. O'Brien, L.E. Strubbe, F. De Colle, E. Ramirez-Ruiz, W.H. Lee, S. Nayakshin, E. Quataert, A.R. King, A. Cucchiara, J. Guillochon, G.C. Bower, A.S. Fruchter, A.N. Morgan, A.J. van der Horst, A Possible Relativistic Jetted Outburst from a Massive Black Hole Fed by a Tidally Disrupted Star. Science 333, 203 (2011). doi:10.1126/science.1207150

C. Bonnerot, D.J. Price, G. Lodato, E.M. Rossi, Magnetic field evolution in tidal disruption events. Mon. Not. R. Astron. Soc. 469(4), 4879-4888 (2017). doi:10.1093/mnras/stx1210

G.C. Brown, A.J. Levan, E.R. Stanway, N.R. Tanvir, S.B. Cenko, E. Berger, R. Chornock, A. Cucchiaria, Swift J1112.2-8238: a candidate relativistic tidal disruption flare. Mon. Not. R. Astron. Soc. 452, 4297-4306 (2015). doi:10.1093/mnras/stv1520

M. Bugli, J. Guilet, E. Müller, L. Del Zanna, N. Bucciantini, P.J. Montero, PapaloizouPringle instability suppression by the magnetorotational instability in relativistic accretion discs. Mon. Not. R. Astron. Soc. 475, 108-120 (2018). doi:10.1093/mnras/stx3158

D.N. Burrows, J.A. Kennea, G. Ghisellini, V. Mangano, B. Zhang, K.L. Page, M. Eracleous, P. Romano, T. Sakamoto, A.D. Falcone, J.P. Osborne, S. Campana, A.P. Beardmore, 
A.A. Breeveld, M.M. Chester, R. Corbet, S. Covino, J.R. Cummings, P. D'Avanzo, V. D'Elia, P. Esposito, P.A. Evans, D. Fugazza, J.M. Gelbord, K. Hiroi, S.T. Holland, K.Y. Huang, M. Im, G. Israel, Y. Jeon, Y.-B. Jeon, H.D. Jun, N. Kawai, J.H. Kim, H.A. Krimm, F.E. Marshall, P. Mészáros, H. Negoro, N. Omodei, W.-K. Park, J.S. Perkins, M. Sugizaki, H.-I. Sung, G. Tagliaferri, E. Troja, Y. Ueda, Y. Urata, R. Usui, L.A. Antonelli, S.D. Barthelmy, G. Cusumano, P. Giommi, A. Melandri, M. Perri, J.L. Racusin, B. Sbarufatti, M.H. Siegel, N. Gehrels, Relativistic jet activity from the tidal disruption of a star by a massive black hole. Nature 476, 421-424 (2011). doi:10.1038/nature10374

J.K. Cannizzo, H.M. Lee, J. Goodman, The disk accretion of a tidally disrupted star onto a massive black hole. Astrophys. J. 351, 38-46 (1990). doi:10.1086/168442

R. Cao, F.K. Liu, Z.Q. Zhou, S. Komossa, L.C. Ho, A large accretion disc of extreme eccentricity in the TDE ASASSN-14li. Mon. Not. R. Astron. Soc. 480(3), 2929-2938 (2018). doi:10.1093/mnras/sty1997

S.B. Cenko, H.A. Krimm, A. Horesh, A. Rau, D.A. Frail, J.A. Kennea, A.J. Levan, S.T. Holland, N.R. Butler, R.M. Quimby, J.S. Bloom, A.V. Filippenko, A. Gal-Yam, J. Greiner, S.R. Kulkarni, E.O. Ofek, F. Olivares E., P. Schady, J.M. Silverman, N.R. Tanvir, D. $\mathrm{Xu}$, Swift J2058.4+0516: Discovery of a Possible Second Relativistic Tidal Disruption Flare? Astrophys. J. 753, 77 (2012). doi:10.1088/0004-637X/753/1/77

C.-H. Chan, J.H. Krolik, T. Piran, Magnetorotational Instability in Eccentric Disks. Astrophys. J. 856(1), 12 (2018). doi:10.3847/1538-4357/aab15c

E.R. Coughlin, M.C. Begelman, Hyperaccretion during Tidal Disruption Events: Weakly Bound Debris Envelopes and Jets. Astrophys. J. 781, 82 (2014). doi:10.1088/0004$637 \mathrm{X} / 781 / 2 / 82$

B. Curd, R. Narayan, GRRMHD simulations of tidal disruption event accretion discs around supermassive black holes: jet formation, spectra, and detectability. Mon. Not. R. Astron. Soc. 483(1), 565-592 (2019). doi:10.1093/mnras/sty3134

L. Dai, A. Escala, P. Coppi, The Impact of Bound Stellar Orbits and General Relativity on the Temporal Behavior of Tidal Disruption Flares. Astrophys. J. Lett. 775, 9 (2013). doi:10.1088/2041-8205/775/1/L9

L. Dai, J.C. McKinney, N. Roth, E. Ramirez-Ruiz, M.C. Miller, A Unified Model for Tidal Disruption Events. Astrophys. J. 859(2), 20 (2018). doi:10.3847/2041-8213/aab429

J.-P. De Villiers, J.F. Hawley, J.H. Krolik, S. Hirose, Magnetically Driven Accretion in the Kerr Metric. III. Unbound Outflows. Astrophys. J. 620(2), 878-888 (2005). doi:10.1086/427142

C. Dotan, N.J. Shaviv, Super-Eddington slim accretion discs with winds. Mon. Not. R. Astron. Soc. 413(3), 1623-1632 (2011). doi:10.1111/j.1365-2966.2011.18235.x

M. Eracleous, M. Livio, J.P. Halpern, T. Storchi-Bergmann, Elliptical Accretion Disks in Active Galactic Nuclei. Astrophys. J. 438, 610 (1995). doi:10.1086/175104

C.R. Evans, C.S. Kochanek, The tidal disruption of a star by a massive black hole. Astrophys. J. Lett. 346, 13-16 (1989). doi:10.1086/185567

P.C. Fragile, P. Anninos, Hydrodynamic Simulations of Tilted Thick-Disk Accretion onto a Kerr Black Hole. Astrophys. J. 623, 347-361 (2005). doi:10.1086/428433

P.C. Fragile, O.M. Blaes, P. Anninos, J.D. Salmonson, Global General Relativistic Magnetohydrodynamic Simulation of a Tilted Black Hole Accretion Disk. Astrophys. J. 668, 417-429 (2007). doi:10.1086/521092

A. Franchini, G. Lodato, S. Facchini, Lense-Thirring precession around supermassive black holes during tidal disruption events. Mon. Not. R. Astron. Soc. 455, 1946-1956 (2016). doi:10.1093/mnras/stv2417

J. Frank, A. King, D.J. Raine, Accretion Power in Astrophysics: Third Edition 2002

E. Gafton, S. Rosswog, Tidal disruptions by rotating black holes: effects of spin and impact parameter. arXiv e-prints (2019)

S. Gezari, S.B. Cenko, I. Arcavi, X-Ray Brightening and UV Fading of Tidal Disruption Event ASASSN-15oi. Astrophys. J. Lett. 851(2), 47 (2017). doi:10.3847/2041$8213 /$ aaa0c2

S. Gezari, R. Chornock, A. Rest, M.E. Huber, K. Forster, E. Berger, P.J. Challis, J.D. Neill, D.C. Martin, T. Heckman, A. Lawrence, C. Norman, G. Narayan, R.J. Foley, G.H. Marion, D. Scolnic, L. Chomiuk, A. Soderberg, K. Smith, R.P. Kirshner, A.G. Riess, S.J. Smartt, C.W. Stubbs, J.L. Tonry, W.M. Wood-Vasey, W.S. Burgett, K.C. Chambers, 
T. Grav, J.N. Heasley, N. Kaiser, R.-P. Kudritzki, E.A. Magnier, J.S. Morgan, P.A. Price, An ultraviolet-optical flare from the tidal disruption of a helium-rich stellar core. Nature 485, 217-220 (2012). doi:10.1038/nature10990

J. Guillochon, E. Ramirez-Ruiz, Hydrodynamical Simulations to Determine the Feeding Rate of Black Holes by the Tidal Disruption of Stars: The Importance of the Impact Parameter and Stellar Structure. Astrophys. J. 767, 25 (2013). doi:10.1088/0004$637 \mathrm{X} / 767 / 1 / 25$

J. Guillochon, E. Ramirez-Ruiz, A Dark Year for Tidal Disruption Events. Astrophys. J. 809, 166 (2015). doi:10.1088/0004-637X/809/2/166

J. Guillochon, M. McCourt, Simulations of Magnetic Fields in Tidally Disrupted Stars. Astrophys. J. Lett. 834(2), 19 (2017). doi:10.3847/2041-8213/834/2/L19

J.F. Hawley, J.H. Krolik, Magnetically Driven Jets in the Kerr Metric. Astrophys. J. 641, 103-116 (2006). doi:10.1086/500385

J.F. Hawley, J.H. Krolik, Sound Speed Dependence of Alignment in Accretion Disks Subjected to Lense-Thirring Torques. Astrophys. J. 866, 5 (2018). doi:10.3847/15384357/aadf90

K. Hayasaki, N. Stone, A. Loeb, Circularization of tidally disrupted stars around spinning supermassive black holes. Mon. Not. R. Astron. Soc. 461, 3760-3780 (2016). doi:10.1093/mnras/stw1387

J.G. Hills, Possible power source of Seyfert galaxies and QSOs. Nature 254, 295-298 (1975). doi:10.1038/254295a0

T.W.-S. Holoien, J.S. Brown, K. Auchettl, C.S. Kochanek, J.L. Prieto, B.J. Shappee, J. Van Saders, The unusual late-time evolution of the tidal disruption event ASASSN15oi. Mon. Not. R. Astron. Soc. 480(4), 5689-5703 (2018). doi:10.1093/mnras/sty2273

T.W.-S. Holoien, M.E. Huber, B.J. Shappee, M. Eracleous, K. Auchettl, J.S. Brown, M.A. Tucker, K.C. Chambers, C.S. Kochanek, K.Z. Stanek, A. Rest, D. Bersier, R.S. Post, G. Aldering, K.A. Ponder, J.D. Simon, E. Kankare, D. Dong, G. Hallinan, N.A. Reddy, R.L. Sanders, M.W. Topping, Pan-STARRS, J. Bulger, T.B. Lowe, E.A. Magnier, A.S.B Schultz, C.Z. Waters, M. Willman, D. Wright, D.R. Young, ASAS-SN, S. Dong, J.L. Prieto, T.A. Thompson, ATLAS, L. Denneau, H. Flewelling, A.N. Heinze, S.J. Smartt, K.W. Smith, B. Stalder, J.L. Tonry, H. Weiland, PS18kh: A New Tidal Disruption Event with a Non-axisymmetric Accretion Disk. Astrophys. J. 880(2), 120 (2019). doi:10.3847/1538-4357/ab2ae1

T. Hung, S.B. Cenko, N. Roth, S. Gezari, S. Veilleux, S. Van Velzen, C.M. Gaskell, R.J Foley, N. Blagorodnova, L. Yan, Discovery of Highly Blueshifted Broad Balmer and Metastable Helium Absorption Lines in a Tidal Disruption Event. arXiv e-prints, 190305637 (2019)

S. Ichimaru, Bimodal behavior of accretion disks: theory and application to Cygnus X-1 transitions. Astrophys. J. 214, 840-855 (1977). doi:10.1086/155314

P.B. Ivanov, V.V. Zhuravlev, J.C.B. Papaloizou, The dynamics of a twisted disc formed after the tidal disruption of a star by a rotating black hole. Mon. Not. R. Astron. Soc. 481, 3470-3496 (2018). doi:10.1093/mnras/sty2493

Y.-F. Jiang, J. Guillochon, A. Loeb, Prompt Radiation and Mass Outflows from the Stream-Stream Collisions of Tidal Disruption Events. Astrophys. J. 830, 125 (2016). doi:10.3847/0004-637X/830/2/125

Y.-F. Jiang, J.M. Stone, S.W. Davis, A Global Three-dimensional Radiation Magnetohydrodynamic Simulation of Super-Eddington Accretion Disks. Astrophys. J. 796, 106 (2014). doi:10.1088/0004-637X/796/2/106

Y.-F. Jiang, S.W. Davis, J.M. Stone, Iron Opacity Bump Changes the Stability and Structure of Accretion Disks in Active Galactic Nuclei. Astrophys. J. 827(1), 10 (2016) doi:10.3847/0004-637X/827/1/10

E. Kara, J.M. Miller, C. Reynolds, L. Dai, Relativistic reverberation in the accretion flow of a tidal disruption event. Nature 535, 388-390 (2016). doi:10.1038/nature18007

E. Kara, L. Dai, C.S. Reynolds, T. Kallman, Ultrafast Outflow in Tidal Disruption Event ASASSN-14li. ArXiv e-prints (2017)

D. Kasen, R.C. Thomas, P. Nugent, Time-dependent Monte Carlo Radiative Transfer Calculations for Three-dimensional Supernova Spectra, Light Curves, and Polarization. Astrophys. J. 651, 366-380 (2006). doi:10.1086/506190 
L.Z. Kelley, A. Tchekhovskoy, R. Narayan, Tidal disruption and magnetic flux capture: powering a jet from a quiescent black hole. Mon. Not. R. Astron. Soc. 445, 3919-3938 (2014). doi:10.1093/mnras/stu2041

M. Kesden, Tidal-disruption rate of stars by spinning supermassive black holes. Phys. Rev. D 85(2), 024037 (2012). doi:10.1103/PhysRevD.85.024037

S. Komossa, Tidal disruption of stars by supermassive black holes: Status of observations. Journal of High Energy Astrophysics 7, 148-157 (2015). doi:10.1016/j.jheap.2015.04.006

J.H. Krolik, T. Piran, Swift J1644+57: A White Dwarf Tidally Disrupted by a $10^{4} M_{\odot}$ Black Hole? Astrophys. J. 743(2), 134 (2011). doi:10.1088/0004-637X/743/2/134

J. Krolik, T. Piran, G. Svirski, R.M. Cheng, ASASSN-14li: A Model Tidal Disruption Event. Astrophys. J. 827(2), 127 (2016). doi:10.3847/0004-637X/827/2/127

W.-H. Lei, B. Zhang, H. Gao, Frame Dragging, Disk Warping, Jet Precessing, and Dipped X-Ray Light Curve of Sw J1644+57. Astrophys. J. 762(2), 98 (2013). doi:10.1088/0004$637 \mathrm{X} / 762 / 2 / 98$

G. Leloudas, L. Dai, I. Arcavi, P.M. Vreeswijk, B. Mockler, R. Roy, D.B. Malesani, S. Schulze, T. Wevers, M. Fraser, E. Ramirez-Ruiz, K. Auchettl, J. Burke, G. Cannizzaro, P. Charalampopoulos, T.-W. Chen, A.a. Cikota, M. Della Valle, L. Galbany, M. Gromadzki, K.E. Heintz, D. Hiramatsu, P.G. Jonker, Z. Kostrzewa-Rutkowska, K. Maguire, I. Mandel, M. Nicholl, F. Onori, N. Roth, S.J. Smartt, L. Wyrzykowski, D.R. Young, The Spectral Evolution of AT 2018dyb and the Presence of Metal Lines in Tidal Disruption Events. Astrophys. J. 887(2), 218 (2019). doi:10.3847/1538-4357/ab5792

D. Lin, J. Guillochon, S. Komossa, E. Ramirez-Ruiz, J.A. Irwin, W.P. Maksym, D. Grupe, O. Godet, N.A. Webb, D. Barret, A likely decade-long sustained tidal disruption event. Nature Astronomy 1, 0033 (2017). doi:10.1038/s41550-016-0033

M. Liska, C. Hesp, A. Tchekhovskoy, A. Ingram, M. van der Klis, S. Markoff, Formation of precessing jets by tilted black hole discs in 3D general relativistic MHD simulations. Mon. Not. R. Astron. Soc. 474(1), 81-85 (2018). doi:10.1093/mnrasl/slx174

M. Liska, A. Tchekhovskoy, A. Ingram, M. van der Klis, Bardeen-Petterson alignment, jets, and magnetic truncation in GRMHD simulations of tilted thin accretion discs. Mon. Not. R. Astron. Soc. 487(1), 550-561 (2019a). doi:10.1093/mnras/stz834

M. Liska, C. Hesp, A. Tchekhovskoy, A. Ingram, M. van der Klis, S.B. Markoff, M. Van Moer, Disc Tearing and Bardeen-Petterson Alignment in GRMHD Simulations of Highly Tilted Thin Accretion Discs. arXiv e-prints, 1904-08428 (2019b)

F.K. Liu, Z.Q. Zhou, R. Cao, L.C. Ho, S. Komossa, Disc origin of broad optical emission lines of the TDE candidate PTF09djl. Monthly Notices of the Royal Astronomical Society: Letters 472(1), 99-103 (2017). doi:10.1093/mnrasl/slx147. https://doi.org/10.1093/mnrasl/slx147

G. Lodato, A.R. King, J.E. Pringle, Stellar disruption by a supermassive black hole: is the light curve really proportional to $\mathrm{t}^{-5 / 3}$ ? Mon. Not. R. Astron. Soc. 392, 332-340 (2009). doi:10.1111/j.1365-2966.2008.14049.x

G. Lodato, D.J. Price, On the diffusive propagation of warps in thin accretion discs. Mon. Not. R. Astron. Soc. 405(2), 1212-1226 (2010). doi:10.1111/j.1365-2966.2010.16526.x

G. Lodato, J.E. Pringle, Warp diffusion in accretion discs: a numerical investigation. Mon. Not. R. Astron. Soc. 381(3), 1287-1300 (2007). doi:10.1111/j.1365-2966.2007.12332.x

G. Lodato, E.M. Rossi, Multiband light curves of tidal disruption events. Mon. Not. R. Astron. Soc. 410(1), 359-367 (2011). http://doi.wiley.com/10.1111/j.13652966.2010.17448.x

A. Loeb, A. Ulmer, Optical Appearance of the Debris of a Star Disrupted by a Massive Black Hole. Astrophys. J. 489, 573-578 (1997). doi:10.1086/304814

W. Lu, P. Kumar, On the Missing Energy Puzzle of Tidal Disruption Events. Astrophys. J. 865(2), 128 (2018). doi:10.3847/1538-4357/aad54a

S.H. Lubow, G.I. Ogilvie, On the Tilting of Protostellar Disks by Resonant Tidal Effects. Astrophys. J. 538, 326-340 (2000). doi:10.1086/309101

S.H. Lubow, J.C.B. Papaloizou, J.E. Pringle, Magnetic field dragging in accretion discs. Mon. Not. R. Astron. Soc. 267(2), 235-240 (1994). doi:10.1093/mnras/267.2.235

J.C. McKinney, Total and Jet Blandford-Znajek Power in the Presence of an Accretion Disk. Astrophys. J. Lett. 630, 5-8 (2005). doi:10.1086/468184

J.C. McKinney, L. Dai, M.J. Avara, Efficiency of super-Eddington magnetically-arrested 
accretion. Mon. Not. R. Astron. Soc. 454, 6-10 (2015). doi:10.1093/mnrasl/slv115

J.C. McKinney, A. Tchekhovskoy, A. Sadowski, R. Narayan, Three-dimensional general relativistic radiation magnetohydrodynamical simulation of super-Eddington accretion, using a new code HARMRAD with M1 closure. Mon. Not. R. Astron. Soc. 441, $3177-$ 3208 (2014). doi:10.1093/mnras/stu762

J.C. McKinney, A. Tchekhovskoy, R.D. Bland ford, General relativistic magnetohydrodynamic simulations of magnetically choked accretion flows around black holes. Mon. Not. R. Astron. Soc. 423(4), 3083-3117 (2012). doi:10.1111/j.1365-2966.2012.21074.x

J.C. McKinney, A. Tchekhovskoy, R.D. Bland ford, Alignment of Magnetized Accretion Disks and Relativistic Jets with Spinning Black Holes. Science 339(6115), 49 (2013). doi:10.1126/science.1230811

D. Merritt, E. Vasiliev, Spin evolution of supermassive black holes and galactic nuclei. Phys. Rev. D 86(10), 102002 (2012). doi:10.1103/PhysRevD.86.102002

B.D. Metzger, N.C. Stone, A bright year for tidal disruptions. Mon. Not. R. Astron. Soc. 461, 948-966 (2016). doi:10.1093/mnras/stw1394

M.C. Miller, Disk Winds as an Explanation for Slowly Evolving Temperatures in Tidal Disruption Events. Astrophys. J. 805, 83 (2015). doi:10.1088/0004-637X/805/1/83

B. Mockler, J. Guillochon, E. Ramirez-Ruiz, Weighing Black Holes Using Tidal Disruption Events. Astrophys. J. 872(2), 151 (2019). doi:10.3847/1538-4357/ab010f

M. Mościbrodzka, H. Falcke, S. Noble, Scale-invariant radio jets and varying black hole spin. Astron. \& Astrophys. 596, 13 (2016). doi:10.1051/0004-6361/201629157

A. Mummery, S.A. Balbus, The spectral evolution of disc dominated tidal disruption events Mon. Not. R. Astron. Soc. 492(4), 5655-5674 (2020). doi:10.1093/mnras/staa192

R. Narayan, I. Yi, Advection-dominated Accretion: A Self-similar Solution. Astrophys. J. Lett. 428, 13 (1994). doi:10.1086/187381

R. Narayan, I.V. Igumenshchev, M.A. Abramowicz, Magnetically Arrested Disk: an Energetically Efficient Accretion Flow. Publications of the Astronomical Society of Japan 55, 69-72 (2003). doi:10.1093/pasj/55.6.L69

R. Narayan, A. Sadowski, R.F. Penna, A.K. Kulkarni, GRMHD simulations of magnetized advection-dominated accretion on a non-spinning black hole: role of outflows. Mon. Not. R. Astron. Soc. 426(4), 3241-3259 (2012). doi:10.1111/j.1365-2966.2012.22002.x

R. Nealon, D.J. Price, C. Bonnerot, G. Lodato, On the Papaloizou-Pringle instability in tidal disruption events. Mon. Not. R. Astron. Soc. 474, 1737-1745 (2018). doi:10.1093/mnras/stx2871

R.P. Nelson, J.C.B. Papaloizou, Hydrodynamic simulations of the Bardeen-Petterson effect. Mon. Not. R. Astron. Soc. 315(3), 570-586 (2000). doi:10.1046/j.13658711.2000.03478.x

C. Nixon, A. King, D. Price, J. Frank, Tearing up the Disk: How Black Holes Accrete. Astrophys. J. Lett. 757(2), 24 (2012). doi:10.1088/2041-8205/757/2/L24

I.D. Novikov, K.S. Thorne, Astrophysics of black holes., in Black Holes (Les Astres Occlus), ed. by C. Dewitt, B.S. Dewitt, 1973, pp. 343-450

G.I. Ogilvie, The non-linear fluid dynamics of a warped accretion disc. Mon. Not. R. Astron. Soc. 304, 557-578 (1999). doi:10.1046/j.1365-8711.1999.02340.x

G.I. Ogilvie, Non-linear bending waves in Keplerian accretion discs. Mon. Not. R. Astron. Soc. 365, 977-990 (2006). doi:10.1111/j.1365-2966.2005.09776.x

G.I. Ogilvie, A.J. Barker, Local and global dynamics of eccentric astrophysical discs. Mon. Not. R. Astron. Soc. 445(3), 2621-2636 (2014). doi:10.1093/mnras/stu1795

K. Ohsuga, S. Mineshige, Global Structure of Three Distinct Accretion Flows and Outflows around Black Holes from Two-dimensional Radiation-magnetohydrodynamic Simulations. Astrophys. J. 736, 2 (2011). doi:10.1088/0004-637X/736/1/2

K. Ohsuga, M. Mori, T. Nakamoto, S. Mineshige, Supercritical Accretion Flows around Black Holes: Two-dimensional, Radiation Pressure-dominated Disks with Photon Trapping. Astrophys. J. 628, 368-381 (2005). doi:10.1086/430728

J.C.B. Papaloizou, D.N.C. Lin, On the dynamics of warped accretion disks. Astrophys. J. 438, 841-851 (1995). doi:10.1086/175127

J.C.B. Papaloizou, J.E. Pringle, The time-dependence of non-planar accretion discs. Mon. Not. R. Astron. Soc. 202, 1181-1194 (1983). doi:10.1093/mnras/202.4.1181

J.C.B. Papaloizou, J.E. Pringle, The dynamical stability of differentially rotating discs with 
constant specific angular momentum. Mon. Not. R. Astron. Soc. 208, 721-750 (1984). doi:10.1093/mnras/208.4.721

D.R. Pasham, R.A. Remillard, P.C. Fragile, A. Franchini, N.C. Stone, G. Lodato, J. Homan, D. Chakrabarty, F.K. Baganoff, J.F. Steiner, E.R. Coughlin, N.R. Pasham, A loud quasi-periodic oscillations after a star is disrupted by a massive black hole. Science $\mathbf{3 6 3}$, 531-534 (2019)

M.E. Pessah, D. Psaltis, The Stability of Magnetized Rotating Plasmas with Superthermal Fields. Astrophys. J. 628, 879-901 (2005). doi:10.1086/430940

E.S. Phinney, Manifestations of a Massive Black Hole in the Galactic Center, in The Center of the Galaxy, ed. by M. Morris IAU Symposium, vol. 136, 1989, p. 543

T. Piran, G. Svirski, J. Krolik, R.M. Cheng, H. Shiokawa, Disk Formation Versus Disk Accretion - What Powers Tidal Disruption Events? Astrophys. J. 806, 164 (2015a). doi:10.1088/0004-637X/806/2/164

T. Piran, A. Sadowski, A.e. Tchekhovskoy, Jet and disc luminosities in tidal disruption events. Mon. Not. R. Astron. Soc. 453(1), 157-165 (2015b). doi:10.1093/mnras/stv1547

P. Polko, J.C. McKinney, Electromagnetic versus Lense-Thirring alignment of black hole accretion discs. Mon. Not. R. Astron. Soc. 464(3), 2660-2671 (2017). doi:10.1093/mnras/stw1875

J. Poutanen, G. Lipunova, S. Fabrika, A.G. Butkevich, P. Abolmasov, Supercritically accreting stellar mass black holes as ultraluminous X-ray sources. Mon. Not. R. Astron. Soc. 377(3), 1187-1194 (2007). doi:10.1111/j.1365-2966.2007.11668.x

D. Proga, J.M. Stone, T.R. Kallman, Dynamics of Line-driven Disk Winds in Active Galactic Nuclei. Astrophys. J. 543(2), 686-696 (2000). doi:10.1086/317154

M.J. Rees, Tidal disruption of stars by black holes of 10 to the 6 th-10 to the 8 th solar masses in nearby galaxies. Nature 333, 523-528 (1988). doi:10.1038/333523a0

M.J. Rees, M.C. Begelman, R.D. Blandford, E.S. Phinney, Ion-supported tori and the origin of radio jets. Nature 295(5844), 17-21 (1982). doi:10.1038/295017a0

R.C. Reis, J.M. Miller, M.T. Reynolds, K. Gültekin, D. Maitra, A.L. King, T.E. Strohmayer, A 200-Second Quasi-Periodicity After the Tidal Disruption of a Star by a Dormant Black Hole. Science 337(6097), 949 (2012). doi:10.1126/science.1223940

N. Roth, D. Kasen, J. Guillochon, E. Ramirez-Ruiz, The X-Ray through Optical Fluxes and Line Strengths of Tidal Disruption Events. Astrophys. J. 827, 3 (2016). doi:10.3847/0004-637X/827/1/3

N. Roth, D. Kasen, What Sets the Line Profiles in Tidal Disruption Events? Astrophys. J. 855(1), 54 (2018). doi:10.3847/1538-4357/aaaec6

A. Sadowski, R. Narayan, Three-dimensional simulations of supercritical black hole accretion discs - luminosities, photon trapping and variability. Mon. Not. R. Astron. Soc. 456, 3929-3947 (2016). doi:10.1093/mnras/stv2941

A. Sadowski, R. Narayan, Powerful radiative jets in supercritical accretion discs around non-spinning black holes. Mon. Not. R. Astron. Soc. 453(3), 3213-3221 (2015). doi:10.1093/mnras/stv1802

A. Sadowski, R. Narayan, A. Tchekhovskoy, Y. Zhu, Semi-implicit scheme for treating radiation under M1 closure in general relativistic conservative fluid dynamics codes. Mon. Not. R. Astron. Soc. 429(4), 3533-3550 (2013). doi:10.1093/mnras/sts632

A. Sadowski, E. Tejeda, E. Gafton, S. Rosswog, D. Abarca, Magnetohydrodynamical simulations of a deep tidal disruption in general relativity. Mon. Not. R. Astron. Soc. 458(4), 4250-4268 (2016). doi:10.1093/mnras/stw589

N.I. Shakura, R.A. Sunyaev, Black holes in binary systems. Observational appearance. Astron. ES Astrophys. 24, 337-355 (1973)

R.-F. Shen, C.D. Matzner, Evolution of Accretion Disks in Tidal Disruption Events. Astrophys. J. 784, 87 (2014). doi:10.1088/0004-637X/784/2/87

H. Shiokawa, J.H. Krolik, R.M. Cheng, T. Piran, S.C. Noble, General Relativistic Hydrodynamic Simulation of Accretion Flow from a Stellar Tidal Disruption. Astrophys. J. 804, 85 (2015). doi:10.1088/0004-637X/804/2/85

K.A. Sorathia, J.H. Krolik, J.F. Hawley, Magnetohydrodynamic Simulation of a Disk Subjected to Lense-Thirring Precession. Astrophys. J. 777(1), 21 (2013a). doi:10.1088/0004$637 \mathrm{X} / 777 / 1 / 21$

K.A. Sorathia, J.H. Krolik, J.F. Hawley, Relaxation of Warped Disks: The Case of Pure 
Hydrodynamics. Astrophys. J. 768(2), 133 (2013b). doi:10.1088/0004-637X/768/2/133

N. Stone, A. Loeb, Observing Lense-Thirring Precession in Tidal Disruption Flares. Physical Review Letters 108(6), 061302 (2012). doi:10.1103/PhysRevLett.108.061302

L.E. Strubbe, E. Quataert, Optical flares from the tidal disruption of stars by massive black holes. Mon. Not. R. Astron. Soc. 400, 2070-2084 (2009). doi:10.1111/j.13652966.2009.15599.x

L.E. Strubbe, E. Quataert, Spectroscopic signatures of the tidal disruption of stars by massive black holes. Mon. Not. R. Astron. Soc. 415(1), 168-180 (2011). doi:10.1111/j.13652966.2011.18686.x

G. Svirski, T. Piran, J. Krolik, Elliptical Accretion and Low Luminosity from High Accretion Rate Stellar Tidal Disruption Events. Mon. Not. R. Astron. Soc. 467(2), 1426-1432 (2017). doi:10.1093/mnras/stx117

D. Syer, C.J. Clarke, The viscous evolution of elliptical accretion discs. Mon. Not. R. Astron. Soc. 255, 92-104 (1992). doi:10.1093/mnras/255.1.92

A. Tchekhovskoy, B.D. Metzger, D. Giannios, L.Z. Kelley, Swift J1644+57 gone MAD: the case for dynamically important magnetic flux threading the black hole in a jetted tidal disruption event. Mon. Not. R. Astron. Soc. 437, 2744-2760 (2014). doi:10.1093/mnras/stt2085

A. Tchekhovskoy, J.C. McKinney, R. Narayan, General Relativistic Modeling of Magnetized Jets from Accreting Black Holes, in Journal of Physics Conference Series. Journal of Physics Conference Series, vol. 372, 2012, p. 012040. doi:10.1088/1742$6596 / 372 / 1 / 012040$

A. Tchekhovskoy, R. Narayan, J.C. McKinney, Efficient generation of jets from magnetically arrested accretion on a rapidly spinning black hole. Mon. Not. R. Astron. Soc. 418(1), 79-83 (2011). doi:10.1111/j.1745-3933.2011.01147.x

L.L. Thomsen, J. Lixin Dai, E. Ramirez-Ruiz, E. Kara, C. Reynolds, X-Ray Fluorescence from Super-Eddington Accreting Black Holes. Astrophys. J. Lett. 884(1), 21 (2019). doi:10.3847/2041-8213/ab4518

M. Toscani, G. Lodato, R. Nealon, Gravitational wave emission from unstable accretion discs in tidal disruption events. Mon. Not. R. Astron. Soc. 489(1), 699-706 (2019). doi:10.1093/mnras/stz2201

A. Ulmer, Flares from the Tidal Disruption of Stars by Massive Black Holes. Astrophys. J. 514, 180-187 (1999). doi:10.1086/306909

S. van Velzen, N.C. Stone, B.D. Metzger, S. Gezari, T.M. Brown, A.S. Fruchter, Late-time UV Observations of Tidal Disruption Flares Reveal Unobscured, Compact Accretion Disks. Astrophys. J. 878(2), 82 (2019). doi:10.3847/1538-4357/ab1844

S. van Velzen, S. Gezari, E. Hammerstein, N. Roth, S. Frederick, C. Ward, T. Hung, S.B. Cenko, R. Stein, D.A. Perley, K. Taggart, J. Sollerman, I. Andreoni, E.C. Bellm, V. Brinnel, K. De, R. Dekany, M. Feeney, R.J. Foley, C. Fremling, M. Giomi, V.Z. Golkhou, A.Y.Q. Ho, M.M. Kasliwal, C.D. Kilpatrick, S.R. Kulkarni, T. Kupfer, R.R. Laher, A. Mahabal, F.J. Masci, J. Nordin, R. Riddle, B. Rusholme, Y. Sharma, J. van Santen, D.L. Shupe, M.T. Soumagnac, Seventeen Tidal Disruption Events from the First Half of ZTF Survey Observations: Entering a New Era of Population Studies. arXiv e-prints, 2001-01409 (2020)

S. Wu, E.R. Coughlin, C. Nixon, Super-Eddington accretion in tidal disruption events: the impactof realistic fallback rates on accretion rates. Mon. Not. R. Astron. Soc. 478(3), 3016-3024 (2018). doi:10.1093/mnras/sty971

M. Xiang-Gruess, P.B. Ivanov, J.C.B. Papaloizou, On the formation of a quasi-stationary twisted disc after a tidal disruption event. Mon. Not. R. Astron. Soc. 463, 2242-2264 (2016). doi:10.1093/mnras/stw2130

J.J. Zanazzi, D. Lai, Tidal Disruption Event Disks around Supermassive Black Holes: Disk Warp and Inclination Evolution. arXiv e-prints (2019)

B.A. Zauderer, E. Berger, A.M. Soderberg, A. Loeb, R. Narayan, D.A. Frail, G.R. Petitpas, A. Brunthaler, R. Chornock, J.M. Carpenter, G.G. Pooley, K. Mooley, S.R. Kulkarni, R. Margutti, D.B. Fox, E. Nakar, N.A. Patel, N.H. Volgenau, T.L. Culverhouse, M.F Bietenholz, M.P. Rupen, W. Max-Moerbeck, A.C.S. Readhead, J. Richards, M. Shepherd, S. Storm, C.L.H. Hull, Birth of a relativistic outflow in the unusual $\gamma$-ray transient Swift J164449.3+573451. Nature 476(7361), 425-428 (2011). doi:10.1038/nature10366 
B.A. Zauderer, E. Berger, R. Margutti, G.G. Pooley, R. Sari, A.M. Soderberg, A. Brunthaler, M.F. Bietenholz, Radio Monitoring of the Tidal Disruption Event Swift J164449.3+573451. II. The Relativistic Jet Shuts Off and a Transition to Forward Shock X-Ray/Radio Emission. Astrophys. J. 767(2), 152 (2013). doi:10.1088/0004$637 \mathrm{X} / 767 / 2 / 152$

Z. Zhu, J.M. Stone, Global Evolution of an Accretion Disk with a Net Vertical Field: Coronal Accretion, Flux Transport, and Disk Winds. Astrophys. J. 857(1), 34 (2018). doi:10.3847/1538-4357/aaafc9 